\title{
Unified Analysis Specific to the Medical Field in the Interpretation of Medical Images through the Use of Deep Learning
}

\author{
Tudor Florin Ursuleanu ${ }^{1,2,3}$, Andreea Roxana Luca ${ }^{1,4}$, Liliana Gheorghe ${ }^{1,5}$, \\ Roxana Grigorovici ${ }^{1}$, Stefan Iancu ${ }^{1}$, Maria Hlusneac ${ }^{1}$, Cristina Preda ${ }^{1,6}$, \\ Alexandru Grigorovici ${ }^{1,2}$
}

\author{
${ }^{1}$ Faculty of General Medicine, “Grigore T. Popa” University of Medicine and Pharmacy, Iasi, Romania \\ ${ }^{2}$ Department of Surgery VI, "Sf. Spiridon” Hospital, Iasi, Romania \\ ${ }^{3}$ Department of Surgery I, Regional Institute of Oncology, Iasi, Romania \\ ${ }^{4}$ Department of Obstetrics and Gynecology, Integrated Ambulatory of Hospital “Sf. Spiridon”, Iasi, Romania \\ ${ }^{5}$ Department of Radiology, "Sf. Spiridon” Hospital, Iasi, Romania \\ ${ }^{6}$ Department of Endocrinology, “Sf. Spiridon” Hospital, Iasi, Romania \\ Email: tudorursuleanu@yahoo.com
}

How to cite this paper: Ursuleanu, T.F., Luca, A.R., Gheorghe, L., Grigorovici, R., Iancu, S., Hlusneac, M., Preda, C. and Grigorovici, A. (2021) Unified Analysis Specific to the Medical Field in the Interpretation of Medical Images through the Use of Deep Learning. E-Health Telecommunication Systems and Networks, 10, 41-74. https://doi.org/10.4236/etsn.2021.102003

Received: May 17, 2021

Accepted: June 21, 2021

Published: June 24, 2021

Copyright $\odot 2021$ by author(s) and Scientific Research Publishing Inc. This work is licensed under the Creative Commons Attribution International License (CC BY 4.0).

http://creativecommons.org/licenses/by/4.0/ (c) (i) Open Access

\section{Abstract}

Deep learning (DL) has seen an exponential development in recent years, with major impact in many medical fields, especially in the field of medical image. The purpose of the work converges in determining the importance of each component, describing the specificity and correlations of these elements involved in achieving the precision of interpretation of medical images using DL. The major contribution of this work is primarily to the updated characterisation of the characteristics of the constituent elements of the deep learning process, scientific data, methods of knowledge incorporation, DL models according to the objectives for which they were designed and the presentation of medical applications in accordance with these tasks. Secondly, it describes the specific correlations between the quality, type and volume of data, the deep learning patterns used in the interpretation of diagnostic medical images and their applications in medicine. Finally presents problems and directions of future research. Data quality and volume, annotations and labels, identification and automatic extraction of specific medical terms can help deep learning models perform image analysis tasks. Moreover, the development of models capable of extracting unattended features and easily incorporated into the architecture of DL networks and the development of techniques to search for a certain network architecture according to the objectives set lead to performance in the interpretation of medical images.
\end{abstract}




\section{Keywords}

Medical Image Analysis, Data Types, Labels, Deep Learning Models

\section{Introduction}

The medical data most used in medical practice are medical images and for this reason most deep learning algorithms have targeted this category of medical information for the realization of medical applications.

This paper presents a methodical review of the literature [1] with the objective of carrying out an analysis of the importance of the relationship between the types and characteristics of scientific data and their use of deep learning models in the interpretation of medical images. We have defined a methodology for semiautomating the production of relevant articles and eliminating those with low impact in the scientific community, by applying inclusive and exclusive quality criteria in the fields of medicine and information technology [2]. The major contribution of this work lies primarily in the updated characterization of the characteristics of the constituent elements of the process of deep learning from data to applications in medicine. Secondly, it describes the specific correlations between data, deep learning models used in the interpretation of diagnostic medical images and their applications in medicine. Finally presents problems and future research directions [3].

The uniqueness of the work is defined by the description of all the constituent elements, namely: data, identification and extraction of automatic standardization of specific medical terms, representation of medical knowledge, incorporation of medical knowledge labeling, description of deep learning (DL) architectures in relation to the objectives for which they were created and in correlation with the other constituent elements of the DL process, presentation of the applications for which they were constituted. Problems in the analysis of the medical image can be classified as follows: identification and extraction and automatic standardization of specific medical terms; representation of medical knowledge; incorporation of medical knowledge. Problems in medical image analysis are related to the following aspects: medical images provided as data for deep learning models require: quality, volume, specificity, labelling; the provision of data from doctors, descriptive data, labels are ambiguous for the same medical and nonstandard references; laborious time in data processing are problems to solve in the future; lack of clinical trials demonstrating the benefits of using DL medical applications in reducing morbidity and mortality and improving patient quality of life [4].

In this paper, we aim to achieve an updated characterization of the specifics of the constituent elements of the deep learning process, scientific data, methods of incorporation of knowledge, DL models according to the objectives for which they were designed and presentation of medical applications according to these 
tasks. Secondly, we will describe the specific correlations between the quality, type and volume of data and their importance in achieving the performance of the deep learning models used in the interpretation of medical diagnostic images [3]. We will also make a structural and functional description of DL models and their applications in medicine.

A large number of medical images are stored in open access databases have private databases of some ceding institutions. These medical images are filed in connection with imaging reports or medical video image reports and, along with language processing from natural images, they have a great contribution to image analysis [5]. Annotation and labelling of the medical image, representing data from doctors, used through methods of integration into deep learning models, consumes time and requires specialized knowledge [3].

The large volume of training data and properly labeled determines the performance of the deep learning modeling in the interpretation of medical images [3]. Because manual image labelling requires time and specialized training, standardized, organized labelling has been used which has the risk of over-labeling with unnecessary information [2].

In the absence of a large amount of data, the problem of over-assembly can be eliminated by adding abandonment. The deep learning model can have increased preformation in these conditions by optimizing a large number of hyper-parameters (size and number of filters, depth, learning rate, activation function, number of hidden layers, etc.) [1] [6].

In medical image analysis the data types have a high variability and can be exemplified by image captures from different regions [7], different types of data included in a phase [8], different types of images [9], data from doctors have errors and require time for processing [10] small sample sizes [11].

A large number of medical images are stored in open access databases have private databases of some ceding institutions. These medical images are filed in connection with imaging reports or medical video image reports and, along with language processing from natural images, they have a great contribution to image analysis [12]. Annotation and labelling of the medical image, representing data from doctors, used through methods of integration into deep learning models, consumes time and requires specialized knowledge.

The large volume of training data and properly labeled determines the performance of the deep learning modeling in the interpretation of medical images. Because manual image labelling requires time and specialized training, standardized, organized labelling has been used which has the risk of over-labeling with unnecessary information [6].

In the absence of a large amount of data, the problem of over-assembly can be eliminated by adding abandonment. The deep learning model can have increased preformation in these conditions by optimizing a large number of hyper-parameters (size and number of filters, depth, learning rate, activation function, number of hidden layers, etc.) [1] [13]. In medical image analysis the data types have 
a high variability and can be exemplified by image captures from different regions [7], different types of data included in a phase [14], different types of images [9], data from doctors have errors and require time for processing [10], small sample sizes [15].

Computer-assisted diagnostics (CAD) in medical imaging and diagnostic radiology through the use of deep learning architectures has progressed to satisfactory results with multiple applications, namely, early detection and diagnosis of breast cancer, lung cancer, glaucoma and skin cancer [3] [16] [17] [18].

The types of images used in the analysis of medical images are: CT, MRI, Xray, Ultra-sound, PET, Wave images, Biopsy, Mammography and Spectrography [1]. In the process of images analysis of the tasks of extracting characteristics, reducing size, augmentation, segmentation, grouping or classification are decisive for the efficiency and precision of integration methods [5] [14] [19] [20].

Larger datasets, compared to the small size of many medical datasets, result in better deep learning models [3] [21].

There are many large-scale and well-annotated data sets, such as ImageNet 1 (over 14 million images tagged in $20 \mathrm{k}$ categories) and COCO 2 (with over 200 images annotated in 80 categories), medical datasets (open source), such as ChestX-ray14 and Deep-Lesion containing medical images tagged over $100 \mathrm{k}$, the others, contain only a few thousand or even hundreds of medical images [3] (Figure 1), and medical applications have developed properly in the medical fields.

The knowledge of experienced clinical-imaging physicians (radiologists, ophthalmologists and dermatologists, etc.) follows certain characteristics in images, namely, contrast, color, appearance, topology, shape, edges, etc., help and are used by deep learning models to perform the main tasks of medical image analysis [3].

The type and volume of medical data, the labels, the category of field knowledge and the methods of their integration into the DL architectures implicitly determine their performance in medical applications.

\section{State of Arts}

The current state of performance of deep learning models and architectures (DL) depends on the nature and quality of the data used in their training. This section shows the data types and DL model description and classification according to medical data types used, objectives and performances in medical applications.

\subsection{Scientific Data and Dataset}

We will further expose, the types of images and medical data used for diagnosis: natural images, medical images, High-level medical data (diagnostic pattern), low-level medical data (areas of images, disease characteristics), manual features used for medical image analysis. 


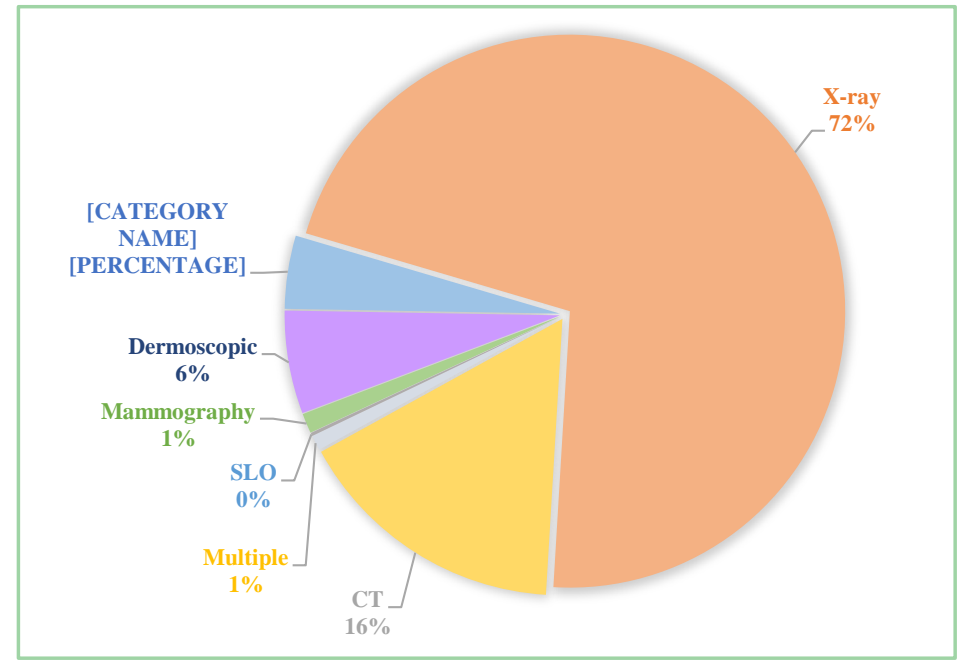

\begin{tabular}{|c|c|c|c|c|}
\hline Imaging & Number of Images & Type & Purpose & Name Datasets \\
\hline Multiple & 1921 patients & Brain & Classification & $\mathrm{ADNI}$ \\
\hline MRI & 539 patients & Brain & Classification & ABIDE \\
\hline MRI & 150 patients & Cardiac & Classification & $\mathrm{ACDC}$ \\
\hline X-ray & $\begin{array}{c}112,120 \text { images - 30,805 } \\
\text { patients }\end{array}$ & Chest & Detection & Chest X-ray14 \\
\hline X-ray & 1018 patients & Lung & Detection & LIDC-IDRI \\
\hline $\mathrm{CT}$ & 888 images & Lung & Detection & LUNA16 \\
\hline $\mathrm{X}$-ray & $\begin{array}{c}40,895 \text { images - } 14,982 \\
\text { patients }\end{array}$ & $\begin{array}{l}\text { Musculo- } \\
\text { skeletal }\end{array}$ & Detection & MURA \\
\hline MRI & 542 images & Brain & Segmentation & BraTS2018 \\
\hline SLO & 400 images & Eye & Segmentation & STARE \\
\hline Mammography & 2500 patients & Breast & $\begin{array}{l}\text { Classification } \\
\text { Detection }\end{array}$ & DDSM \\
\hline $\mathrm{CT}$ & $\begin{array}{l}32,735 \text { images }-4427 \\
\text { patients }\end{array}$ & Multiple & $\begin{array}{l}\text { Classification } \\
\text { Detection }\end{array}$ & Deep-Lesion \\
\hline MRI & 7980 images - 33 cases & Cardiac & $\begin{array}{l}\text { Classification } \\
\text { Segmentation }\end{array}$ & Cardiac MRI \\
\hline Dermoscopy & 13,000 images & Skin & $\begin{array}{l}\text { Classification } \\
\text { Detection } \\
\text { Segmentation }\end{array}$ & ISIC 2018 \\
\hline
\end{tabular}

Figure 1. Types of medical images and datasets. Acronyms: MRI Magnetic Resonance Images, CT Computed Tomography, SLO Scanning Laser Ophthalmoscopy images, The alzheimer's disease neuroimaging initiative (ADNI), Automated cardiac diagnosis challenge (ACDC), The autism brain imaging data exchange (ABIDE), Hospital-scale chest X-ray database and benchmarks on weakly-supervised classification and localization of common thorax diseases (Chestx-ray14), The lung image database consortium (lidc) and image database resource initiative (idri) (LIDC-IDRI), Algorithms for automatic detection of pulmonary nodules in computed tomography images (LUNA16), Large dataset for abnormality detection in musculoskeletal radiographs (MURA) [3], Machine learning algorithms for brain tumor segmentation, progression assessment, and overall survival prediction in the brats challenge (BraTS2018) [3], Locating blood vessels in retinal images (STARE), Digital database for screening mammography (DDSM), Automated mining of large-scale lesion annotations and universal lesion detection with deep learning (DeepLesion), Cardiac Magnetic Resonance Images (Cardiac MRI), International skin imaging collaboration (ISIC). 
Natural images-from natural datasets, ImageNet 1 (over 14 million images tagged in $20 \mathrm{k}$ categories) and COCO 2 (with over 200 images annotated in 80 categories). Large natural images (ImageNet) are incorporated for the detection of objects in the medical field and are used in applications for the detection of lymph nodes [22], detection of polyp and pulmonary embolism [23], detection of breast tumors [24], detection of colorectal polyps [25] [26]. Natural Images, ImageNet, PASCAL VOC "static data" set, Sports-1M video datasets, which is the largest video classification indicator with 1.1 million sports videos in 487 categories [3] [27].

Medical images from external medical datasets of the same diseases in similar ways (e. g. SFM and DM) [28], medical images from external medical datasets of the same diseases [3] in different ways (DBT and MM, ultrasound) [29] or from different diseases [30]. Medical images are used in multiple applications. Multi-modal medical images, PET images are incorporated for the detection of lesions in CT scans of the liver [31]. Multimodal medical images are also used in another model in the detection of liver tumors [32]. Multimodal medical images (mammographic data) are used to detect breast masses [33]. Medical images, (CT, MRI, angio-CT, butt eye images), annotated retinal images, used to help segment the heart vessel without annotations [3] [34]. External medical data and images of other diseases, such as the union dataset (3DSeg-8) by aggregating eight sets [3] of 3D medical segmentation data [35].

Medical data from doctors: high-level medical data (diagnostic pattern) and low-level medical data (areas of images, disease characteristics). High-level and low-level medical data, i.e. anatomical aspects of the image, shape, position, typology of lesions integrated into segmentation tasks, example of the ISBI 2017 dataset used in skin injury segmentation. The use of additional medical datasets in different ways has also proven to be useful, although most applications are limited in using MRI to help segmentation tasks in CT images [3] [36]. Specific data identified by doctors (attention maps, hand-highlighted features) increase the diagnostic performance of deep learning networks (no comparative studies have been conducted). Medical data from doctors, handmade features, handcrafted features, invariant LBP, as well as $\mathrm{H}$ \& Components, are calculated first from the images [3]. The use of the BRATS2015 data set in applications in which these features are used is achieved performance in image segmentation by input-level fusion. However, anatomical priorities are only suitable for segmentation of fixed-shaped organs [3] such as the heart or lungs [35].

Manual features used for medical image analysis is a series of measurements (X-ray projections in CT or spatial frequency information in MRI). The methods based on deep learning have been widely applied in this area [37] [38]. Examples: image reconstruction with optical diffuse tomography (DOT), reconstruction of magnetic resonance imaging by compressed detection (CS-MRI) [39], reconstruction of the image with diffuse optical tomography (DOT) of limitedangle breast cancer and limited sources in a strong scattering environment [40], 
recovery of brain MRI images, target contrast using GAN. Content-based image recovery (CBIR) can be great help to for the clinicians to navigate these large data sets. Some deep learning methods [3] adopt transfer learning to use knowledge from natural images or external medical datasets [41] [42] [43], for example, metadata such as age and sex of patients, characteristics extracted from health areas, decision values of binary traits and texture traits in the process of thoracic X-ray recovery [3].

Medical data used to generate medical reports, subtitling medical images, templates from radiologist reports, visual characteristics of medical images, generating reports using the IU-RR dataset.

\subsection{Addressing Label Noise in the Formation of Deep Learning Patterns in Medical Image Analysis}

The noise of the label in the formation of deep learning models is important in their performance for medical image analysis. The approach of the label noise was achieved by: cleaning and pre-processing labels, improving the network architecture with noise layer, the endowment of networks with loss functions, data re-weighting, data and label consistency, training procedures.

Cleaning and pre-processing labels

In chest X-ray scans in the classification of thoracic diseases, the smoothing of labels was used to handle noisy labels and led to improvements of up to 0.08 in the area below the characteristic receptor operating curve (ASC) [44].

Network Architectures

In the case of network architectures, the noise layer proposed by [45] improved the accuracy in detecting breast lesions in mammograms.

Loss functions

The enhancement of networks with loss functions that cause annotations to dilate with a small and large structuring element to generate noisy masks for the foreground and background, e.g. parts of the ring union image were marked as unsafe regions that were ignored during training [46].

Re-weighting data

The method of re-weighting data to cope with noisy annotations in cancer detection was achieved by training models on a large group of noisy label patches using calculated features from a small set of clean label patches and increased model performance by $10 \%$. [47]. This strategy was used to classify skin lesions in noisy label images [48], for segmentation of the heart, clavicles and lung in chest X-rays [10], for segmenting the skin lesion from highly inaccurate annotations [49] proposed a specific characteristic of pixels.

Consistency of data and labels

For segmentation of the left atrium in THE MRI from tagged and unlabeled data it was proposed to form two separate models: a teacher model that produced noisy labels and labeled maps with non-certainties on unlabeled images and a student model that was trained using the noisy labels generated, while 
taking into account the uncertainty of the label and making correct predictions on the clean data set in accordance with the teacher's model on the label, with uncertainty below the threshold.

Training procedures

For segmentation of the bladder, prostate and rectum in MRI, a model was trained on a clean label data set and used it to predict segmentation masks for a separate set of unlabeled data, and a second model was instructed to estimate a confidence map to indicate regions where predicted labels were more likely to be accurate and reliable paper used to sample the main model with a $3 \%$ improvement in the Dice similarity coefficient (DSC) [50]. A rather similar method has been used to classify aortic valve defects in MRI [51].

\subsection{Model Description and Classification According to Medical Data Types Used, Objectives and Performances in Medical Applications}

We will synthesize in Figure 2 classification of DL models according to the characteristics and tasks for which they were designed, classification of DL models according to the characteristics and tasks for which they were designed.

DL architectures can be divided into three categories: [1]

- Supervised

- Unsupervised

- Semi-supervised Supervised DL models: [1]

- Recurent neural networks (RNN), short-term memory (LSTM), closed recurring unit (GRU),

- Convolutional neural networks (CNN) and

- Network of generational opponents (GAN). Unsupervised deep learnirng models: [1]

- Deep Faith Networks (DBN),

- Deep Transfer Network (DTN),

- Tensor Deep Stack Networks (TDSN),

- Autoencoders (AE). [1]

\subsubsection{Below We Describe the DL Models}

$\mathrm{CNN}$ (convolutional neural network) are popular in areas where the shape of an object is an important feature, such as image analysis [4] [52] [53] [54] [55] [56], particularly in the study of cancers and bodily injuries in the medical sector [57] [58] and video analysis [4] [59].

CNN contains convolutive layers, grouping layers, dropout layers, and an output layer, hierarchically positioned that each learn stun specific characteristics in the image [14].

$\mathrm{CNN}$ in image analysis has low performance when high-resolution datasets are considered [60] and when localization over large patches is required, especially in medical images [61]. 


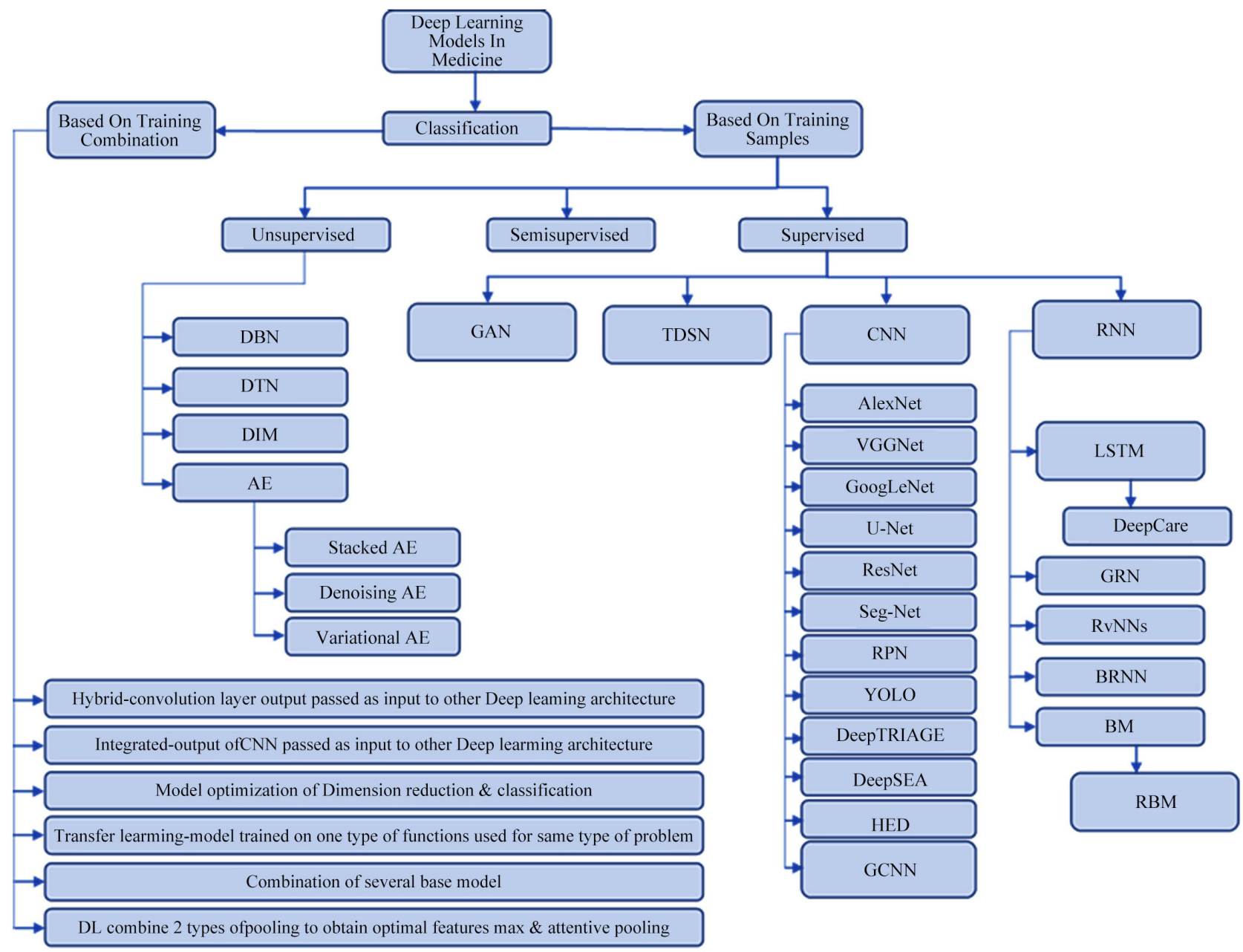

Figure 2. Classification of DL models according to the characteristics and tasks for which they were designed. Acronyms: Deep Network of Beliefs (DBN), Deep Network of Distribution and Target, Deep Info Max (DIM), AutoEnconder (AE), Generative Adversarial Network (GAN), Tensor Deep Stacking Network (TDSN), Convolutional Neural Network (CNN), Visual Geometry Group Network (VGG Net), Deep Layers Network (GoogLeNet), Fully Convolutional Network (U-Net), Residual Neural Network (ResNet), Deep Segmentation-Emendation Network (SegNet), Region Proposal Net (RPN), You Only Look Once (YOLO), Deep Triage (DT), deep learning-based algorithmic framework (DeepSEA), Holistically-Nested Edge Detection (HED), Graph Convolutional Natural Net (GCNN), Recurent Neuronal Network (RNN), Deep Dynamic Neural Network (Deep Care), Gated Recurrent Network (GRN), Recurrsive RNN (RvNNs), Long Short-Term Memory (LSTM), Bidirectional RNN (BRNN), Restricted Boltzmann Machine (RBM).

Image analysis performance is enhanced by the use of the following architectures: AlexNet, VGGNet and ResNet, YOLO or U-net that we describe below:

AlexNet was proposed by [58] [59] for the ImageNet Large Scale Visual Recognition Challenge (ILSVRC) in 2012 [4].

AlexNet consists of 8 layers, 5 layers of convolution and 3 dense, fully connected layers, overlapping overlay, abandonment, data augmentation, ReLU activations after each convolutive layer and fully connected, SGD with impulse [1] [62]. AlexNet is used for image recognition in image analysis and is usually applied to issues involving semantic segmentation and high-resolution data classification tasks [63] [64]. 
VGG (Visual Geometry Group): Consists of 13 convolution layers (in VGG16) \& 16 convolution layers (in VGG19), 3 dense layers, pooling and three RELU units, very small responsive fields [1] [65]. VGG is used for object recognition, classification of medical images [66] [67] and image segmentation [68]. VGG loses accuracy when the depth becomes too high.

ResNet (Residual Neural Network): Contains closed units or closed recurring units and has a strong similarity to recent successful elements applied in RNNs [1]. ResNet is characterized by: residual mapping, identity function, and a twolayer residual block, one layer learns from the residue, the other layer learns from the same function and has high level of performance in image classification (Saravanan et al., Saravanan) and audio analysis tasks [4] [69].

GoogLeNet is built from 22 deep LAYERS CNN and 4 million parameters and contains several layer filters and stacked convolution layers [70] It was used for batch normalization, image distortions, and RMSprop [1].

U-Net, developed by Ronneberger [4] [61], addresses the problem of locating images of a standard CNN by extracting data features followed by reconstruction of the original dimension through an up-sampling operation. U-Net is a type of Enconder-Decoder network in which the codoficator output belongs to the input space [4]. U-Net is used in single-stage segmentation and classification [71], specifically in the locatio;n of cancerous lesions [72] [73] [74]. SegNet [75] is a UNet variant that uses maximum grouping indices in the upsampling step that reduces the complexity of U-Net space.

RNNs were developed by Rumelhart et al. [4] [76] using with efficiency the correlations existing between input data of a prediction problem, through which they process sequential data in relation to text analysis [77] [78] [79], in electronic medical records to predict diseases [4] [80] [81] and speech recognition [82]. RnN variants are: one-way, learning from the past and predicting the future and bidirectional that uses the future to restore the past. RNN has the following variants: short-term memory (LSTM) and closed recurring units (GRU), recursive neural networks (Recursive NNs), two-way RNNs (BiRNN). Shortterm memory LSTMs were introduced by [4] [67] [83] and consist of: the gate of oblivion that alleviates the escape and explosion gradient, the entrance gate and the exit gate, the last two track the flow of data coming in and out of the cell. They were used in speech recognition [84], path prediction [85] and medical diagnosis [86], in which the authors proposed an LSTM network, called DeepCare, combining different types of data to identify clinical diseases.

GURs (recurring unit gated) created by [87] [88] solve the problem of increasing the time complexity of LSTM, when large amounts of data are used [4]. The GRU consists of a reset gate in which it is decided how much information from the past is transmitted in the future, and an update gate that decides how much information from the past can be forgotten. GRU and LSTMs have similar applications especially in speech recognition [89].

The two-way recurring neural network and the Boltzmann BRNNs [4] intro- 
duced by [90] [91] are characterized by the fact that the hidden state is updated by using past information, as in a classic RNN, and by using information related to future moments [4]. They were applied in handwriting and speech recognition, where they are used to detect missing parts of a sentence in a knowledge of the other words [92] [93].

BM models, introduced by [94] [95], are a family of RNNs that are easy to implement and that reproduce many probability distributions, BMs are used in image classification [4]. BMs combined with other models are used to locate objects, [96] [97]. In the classification of images, BMs are used to identify the presence of a tumor [98]. BM models are slow and ineffective when the data size increases exponentially due to the complete connection between neurons [99]. A restricted BM was proposed in which relaxing the connections between neurons of the same or one-way connection between neurons would solve the problem of the classic BM model [100].

AEs, developed by [101] [102], consisting of encoder and decoder, with the aim of reducing the size of the data through significant representations and learning data characteristics for the reconstruction of outputs. They are used in applications in medical image analysis [4] [103], natural language processing [104] and video analysis [105].

Additional variants of $\mathrm{AE}$ that can be found in the literature are variational $\mathrm{AE}$ (VAE). In a VAE, the encoder is represented by the probability density function of the input into the feature space and, after the encoding stage, a sampling of the new data using the PDF is added. Differently from the DAE and the SAE, a VAE is not a regularized $\mathrm{AE}$, but is part of the generation class [4].

GAN it is used to generate synthetic training data from original data using latent distribution [1] [106]. It consisted of two networks, a generator estimates false data from input data, and a discriminator, which differentiates fake data from real data and separates it in order to increase the quality of the data generated. GAN has two problems: the problem of the collapse of the mode, and the fact that, can become very unstable.

The DBN (Deep Network of Beliefs), created by Hinton [107], consists of two networks that build each other: of beliefs represented by an acyclic graph composed of layers of stochastic binary units with weighted and respectively weighted connections, restricted Boltzmann Machines which is a stochastic [1]. DBNs are applied in image recognition and speech recognition, in classification to detect lesions in medical diagnosis and, in video recognition to identify the presence of persons [108], in speech recognition to understand missing words in a sentence [109] and in application on physiological signals to recognize human emotion [110].

DTN contains a characteristic extraction layer, which teaches a shared feature subspace in which marginal source distributions and target samples are drawn close and a layer [1] of discrimination that match conditional distributions by classified transduction [111]. It is used for large-scale problems [1]. 
TDSN contains two parallel hidden representations that are combined using a bilinear mapping [1] [112]. This arrangement provides better generalization compared to the architecture of a single module. The prejudices of the generalisers with regard to the learning set shall be inferred. It works effectively and better than an eco-validation strategy when used with multiple generalisers compared to individual generalisers [1].

Deep InfoMax (DIM): Maximizes mutual information between an input and output of a highly flexible convolutive encoder [113] by forming another neural network that maximizes a lower limit on a divergence between the marginal product of encoder input and output. Estimates obtained by another network can be used to maximize the reciprocal information of the features in the input encoder. The memory requirement of the DIM is lower because it requires only encoder not decoder [1].

\subsubsection{Combinations of Different DL Models Depending on the Type of Data Involved in the Problem to Be Solved}

DL models can be combined in five different ways depending on the type of data involved in the problem to be solved [1] [4]. Of these, three types of HA (hybrid architectures), namely the integrated model, the built-in model and the whole model.

In the integrated model, the output of the convolution layer is transmitted directly as input to other architectures to the residual attention network, the recurrent convolutive neural network (RCNN) and the model of the recurrent residual convolutive neural network (IRRCNN) [114].

In the built-in model (the improved common hybrid CNNBiLSTM), the size reduction model and the classification model perform together, the results of one represent the inputs for the other model. In the model (EJH-CNN-BiLTM), several basic models are combined.

In the learning transfer model (TL) is trained and uses the same type of problem. CNN models that use the TL model are VGG (e.g. VGG16 or VGG19), GoogLeNet (e.g. InceptionV3), Inception Network (Inception-v4), Repiuled Neural Network (e.g. ResNet50), AlexNet. Joint AB based DL combines max pooling, and careful sharing [1].

\subsubsection{Combinations of Different DL Models to Benefit from the Characteristics of Each Model with Medical Applications Are: $\mathrm{CNN}+\mathrm{RNN}, \mathrm{AE}+\mathrm{CNN}$ and GAN + CNN}

$\mathrm{CNN}+\mathrm{RNN}$ are used for the capabilities of the CNN feature extraction model and the RNNs [15]. Because the result of a CNN is a $3 \mathrm{D}$ value and an RNN works with 2D-data, a remodeling layer is, associated between CNN and RNN, to convert THE production of CNN into an array [4]. CNN + RNN have been successfully applied in text analysis to identify missing words [115] and image analysis to increase the speed of magnetic resonance image storage [116] [117]. $\mathrm{CNN}+\mathrm{RNN}$ variants are obtained by replacing the Standard RNN component [4] with an LSTM component [24] [118]. 
The $\mathrm{AE}+\mathrm{CNN}$ architecture combines $\mathrm{AE}$ as a pre-training model when using data with high noise levels, and a CNN as a feature extractor model [4]. AE + NVs have an application in image analysis to classify noisy medical images [119] and in the reconstruction of medical images [120] [121].

GAN + CNN combines GAN as a pre-workout model to moderate the problem of over-mounting, and a CNN, used as a feature extractor [4]. It has applications in image analysis [11] [122].

The DL architectures applied especially in image analysis are CNN, AE and GAN. NVs preserve the spatial structure of the data, and are used as feature extractors (especially U-Net), AEs reduce the characteristics of complex images in the analysis process, and GANs are pre-training architectures that select input categories to control overfitting.

\subsection{Applications in Medicine and the Performance of DL Models Depending on the Therapeutic Areas in Which They Were Used}

We further highlight the acquisitions in the study of deep learning and its applications in the analysis of the medical image, between 2017 and 2020 [4]. You can easily identify references to image labeling and annotation, developing new deep learning models with increased performance, and new approaches to medical image processing:

- diagnosis of cancer by using CNN with different number of layers [123],

- studying deep learning optimization methods and applying in the analysis of medical images [124],

- development of techniques used for endoscopic navigation [125],

- highlighting the importance of data labelling and annotation and knowledge of model performance [126] [127],

- perfecting the layer-wise architecture of convolution networks [1], lesson the cost and calculation time for processor training [128],

- description of the use of AI and its applications in the analysis [1] of medical images [129],

- diagnosis in degenerative disorder using depp learning techniques [130] and,

- detection of cancer by processing medical images using the medium change filter technique [131],

- classification of cancer using histopathological images and highlighting the rapidity of Theano, superior tensor flow [131],

- development of two-channel computational algorithms using DL (segmentation, extraction of characteristics, selection of characteristics and classification and classification, extraction of high-level captures respectively) [132],

- malaria detection using a deep neural network (MM-ResNet) [8].

We will exemplify in Table 1 [2] applications in medicine and the performance of DL models depending on types of medical images and the therapeutic areas in which they were used. We included most relevant papers about the most used medical investigations, respectively medical images. 
Table 1. Applications and the performances of the DL models depending on the types of medical images and the therapeutic area [2]. Acronyms: AMD, age-related Macular Degeneration, CAD, Computer Aided Diagnosis, CNN, Con-volutional Neural Network, MRI, Magnetic Resonance Images, PET, Photon Emission Tomogra-phy, CT, Computed Tomography, OCT, Optical Coherence Tomography, D, dimensions, AUC, Area Under the Curve, MSE, Mean Squared Error, RMSE, Root Mean Square Error, DSC, Dice Similarity Coefficient [2].

\begin{tabular}{|c|c|c|c|c|c|c|c|}
\hline & Type of Data & Sample & Objective & Model Design & Results & Therapeutic Area & Paper \\
\hline & $\begin{array}{l}\text { Mammography } \\
\text { images }\end{array}$ & 45,000 images & $\begin{array}{l}\text { Detect malign solid } \\
\text { lesions and prevent } \\
\text { overtreatment in false } \\
\text { positives [2] }\end{array}$ & $\mathrm{CNN}$ & AUC of 0.90 & Oncology & [89] \\
\hline & Mammography & $\begin{array}{l}667 \text { benign and } \\
333 \text { malignant }\end{array}$ & $\begin{array}{l}\text { Mammography } \\
\text { diagnosis of early } \\
\text { malignant breast }\end{array}$ & Stacked AE & Accuracy of 0.89 & Oncology & {$[96]$} \\
\hline & $\begin{array}{l}\text { Digital } \\
\text { Mammography } \\
\text { images and the } \\
\text { biopsy result of } \\
\text { the lesions [2] }\end{array}$ & $\begin{array}{l}1000 \text { malignant } \\
\text { masses and } 600 \\
\text { cysts images and } \\
\text { their biopsy [2] }\end{array}$ & $\begin{array}{l}\text { Discriminate benign } \\
\text { cysts from malignant } \\
\text { masses }\end{array}$ & $\mathrm{CNN}$ & AUC of 0.80 & Oncology & [97] \\
\hline \multirow[t]{5}{*}{ Mammography } & $\begin{array}{l}\text { Mammography } \\
\text { images }\end{array}$ & $\begin{array}{l}840 \text { images of } \\
\text { mammograms } \\
\text { from } 210 \text { different } \\
\text { patients }\end{array}$ & $\begin{array}{l}\text { Breast arterial } \\
\text { calcification on } \\
\text { mammograms } \\
\text { classifier to evaluate } \\
\text { the risk of coronary } \\
\text { disease [2] }\end{array}$ & $\mathrm{CNN}$ & $\begin{array}{l}\text { Misclassfied cases } \\
\text { of } 6 \%\end{array}$ & Cardiovascular & {$[101]$} \\
\hline & $\begin{array}{l}\text { Digital } \\
\text { mammograms }\end{array}$ & $\begin{array}{l}661 \text { from } 444 \\
\text { patients }\end{array}$ & $\begin{array}{l}\text { Computer automated } \\
\text { estimation of breast } \\
\text { percentage density [2] }\end{array}$ & $\mathrm{CNN}$ & AUC of 0.981 & Oncology & {$[151]$} \\
\hline & $\begin{array}{l}\text { Mammography } \\
\text { images }\end{array}$ & $\begin{array}{l}\text { Mammograms } \\
\text { from } 604 \\
\text { women }\end{array}$ & $\begin{array}{l}\text { Segment areas of dense } \\
\text { fibroglandular tissue in } \\
\text { the breast [2] }\end{array}$ & $\mathrm{CNN}$ & Accuracy of 0.66 & Oncology & {$[116]$} \\
\hline & $\begin{array}{l}\text { Digital } \\
\text { mammograms } \\
\text { images }\end{array}$ & $\begin{array}{l}29,107 \text { left } \\
\text { mediolateral } \\
\text { oblique, right } \\
\text { mediolateral } \\
\text { oblique, left } \\
\text { cranial-caudal } \\
\text { and right } \\
\text { cranial-caudal } \\
\text { mammograms } \\
\text { images }\end{array}$ & $\begin{array}{l}\text { Probability of cancer } \\
\text { on mammograms [2] }\end{array}$ & $\mathrm{CNN}$ & AUC of 0.90 & Oncology & {$[121]$} \\
\hline & $\begin{array}{l}\text { Image of the } \\
\text { heart } 2 \mathrm{D}\end{array}$ & $\begin{array}{l}400 \text { images with } \\
\text { five different heart } \\
\text { diseases and } 80 \\
\text { normal } \\
\text { echocardiogram } \\
\text { images }\end{array}$ & $\begin{array}{l}\text { Segment left ventricle } \\
\text { images with greater } \\
\text { precision }\end{array}$ & $\begin{array}{l}\text { Deep belief } \\
\text { networks }\end{array}$ & $\begin{array}{l}\text { Hammoude } \\
\text { distance of } 0.80\end{array}$ & Cardiovascular & {$[152]$} \\
\hline \multirow[t]{2}{*}{ Ultrasound } & $\begin{array}{l}\text { Ultrasound } \\
\text { imaging }\end{array}$ & $\begin{array}{l}306 \text { malignant and } \\
136 \text { benign tumors } \\
\text { images }\end{array}$ & $\begin{array}{l}\text { CAD system to detect } \\
\text { and differentiate breast } \\
\text { lesions with ultrasound }\end{array}$ & $\begin{array}{l}\text { CNNs inspired in } \\
\text { AlexNet, U-Net } \\
\text { and LeNet }\end{array}$ & $\begin{array}{l}\text { Best F-measure of } \\
0.91 \text { and } 0.89 \\
\text { depending on the } \\
\text { data }\end{array}$ & Oncology & [2] [24] \\
\hline & $\begin{array}{l}\text { Transesophageal } \\
\text { ultrasound } \\
\text { volume and 3D } \\
\text { geometry of the } \\
\text { aortic valve } \\
\text { images }\end{array}$ & $\begin{array}{l}3795 \text { volumes from } \\
\text { the aortic valves } \\
\text { from } 150 \text { patients }\end{array}$ & $\begin{array}{l}\text { Diagnose, stratification } \\
\text { and treatment planning } \\
\text { for patients with aortic } \\
\text { valve pathologies }\end{array}$ & $\begin{array}{l}\text { gMarginal space } \\
\text { deep learning }\end{array}$ & $\begin{array}{l}\text { Position error of } \\
1.66 \mathrm{mms} \text { and } \\
\text { mean corner } \\
\text { distance error of } \\
3.29 \mathrm{mms}\end{array}$ & Cardiovascular & [2] [84] \\
\hline
\end{tabular}




\section{Radiography \\ images}

Radiography

images

\section{Radiography}

Radiography
images

\section{2,120 frontal}

view chest

radiographs from

30,805 patients and

17,202 frontal view

chest radiographs

Abnormality detection $\mathrm{CNN}$

with a binary class

label for normal vs

abnormal

$\begin{array}{ll}\text { Pathology cancer } & \\ \text { images } & \text { tumor images } \\ \text { (hematoxylin and } & \text { tumor infiltrating } \\ \text { eosin) } & \text { lymphocytes }\end{array}$

Study of tumor tissue

samples. Localize areas

of necrosis and

Two CNNs

lymphocyte infiltration

Giemsa-stained 27,558 cell images

thin blood smear 150 infected and

slides cell images 50 healthy patients

Create a screening

system for Malaria

CNN

AUROCs of 0.960

and 0.951 .

AUROCs of 0.900 Radiology

and 0.893

AUC of $0.95 \quad$ Oncology

[2];

Accuracy of 0.94 Infectious Disease [80]

Accuracy of 0.77

for four class

classification and

Microscopy

249 images

image patches

belonging to 20

histologic

Classification of

CNN with a

breast cancer histology Support Vector

categories

microscopy images

Machine (SVM)

an accuracy of 0.83 Oncology

for carcinoma

/noncarcinoma

classification

CAD for breast

Microscopy 7909 images of

histopathological eight subclasses of

images

breast cancers

cancer

histopathological

CNN

Accuracy of 0.93 Oncology

diagnosis

Microscope

images

Whole-slide

prostate

histopathology

images

2D Ocular
fundus images $\quad 243$ retina images $\begin{aligned} & \begin{array}{l}\text { Diagnose retinal } \\ \text { lesions }\end{array}\end{aligned}$

200 female subjects Cervix cancer

aged from 22 to 64 screening

2663 images from

32 whole slide

prostate

histopathology

images

Whole-slide histopathology images

to outline the

CNN

Multiscale CNN

deviation of 0.95 Oncology

and 0.18

Dice coefficient of

0.72

Oncology

[2];

Precision recall

curve of 0.86 in

microaneurysms Ophthalmology

and 0.64 in

[2];

CNN

exudates

$\begin{array}{ll}\text { Ocular fundus } & \text { Over 85,000 } \\ \text { images 2D } & \text { images }\end{array}$

Diabetic retinopathy

detection and stage Bayesian CNN classification
AUC value of 0.99 Ophthalmology 


\begin{tabular}{|c|c|c|c|c|c|c|c|}
\hline & $\begin{array}{l}\text { Color ocular } \\
\text { fundus images }\end{array}$ & $\begin{array}{l}6679 \text { random } \\
\text { sampling images } \\
\text { from Kaggle's } \\
\text { Diabetic } \\
\text { Retinopathy } \\
\text { Detection }\end{array}$ & $\begin{array}{l}\text { Detect retinal } \\
\text { hemorrhages }\end{array}$ & CNN & $\begin{array}{l}\text { AUC of } 0.894 \text { and } \\
0.972\end{array}$ & Ophthalmology & [95] \\
\hline & $\begin{array}{l}\text { Ocular fundus } \\
\text { images }\end{array}$ & $\begin{array}{l}168 \text { images with } \\
\text { glaucoma and } 428 \\
\text { control }\end{array}$ & $\begin{array}{l}\text { System to detect and } \\
\text { evaluate glaucoma }\end{array}$ & $\begin{array}{l}\text { CNN: ResNet and } \\
\text { U-Net }\end{array}$ & $\begin{array}{l}\text { AUC of } 0.91 \text { and } \\
0.84 \text { respectively }\end{array}$ & Ophthalmology & [98] \\
\hline & $\begin{array}{l}\text { Ocular fundus } \\
\text { images }\end{array}$ & $\begin{array}{l}90,000 \text { images with } \\
\text { their diagnoses }\end{array}$ & $\begin{array}{l}\text { Predict the evolution of } \\
\text { diabetic retinopathy } \\
\text { with fundus images }\end{array}$ & CNN & AUC of 0.95 & Ophthalmology & [155] \\
\hline & Fundus images & $\begin{array}{l}7000 \text { colour fundus } \\
\text { images }\end{array}$ & $\begin{array}{l}\text { Image quality in the } \\
\text { context of diabetic } \\
\text { retinopathy }\end{array}$ & CNN & Accuracy of $100 \%$ & Ophthalmology & [156] \\
\hline & $\begin{array}{l}\text { AREDS (age } \\
\text { related eye disease } \\
\text { study) image }\end{array}$ & $\begin{array}{l}\text { e } \\
\text { images }\end{array}$ & $\begin{array}{l}\text { Diagnosis of } \\
\text { Age-related Macular } \\
\text { Degeneration }\end{array}$ & CNN & $\begin{array}{l}94.97 \text { sensitivity } \\
\text { and } 98.32 \% \\
\text { specificity }\end{array}$ & Ophthalmology & [157] \\
\hline & Fundus images & $\begin{array}{l}219,302 \text { from } \\
\text { normal } \\
\text { participants } \\
\text { without } \\
\text { hypertension, } \\
\text { diabetes mellitus } \\
\text { (DM), and any } \\
\text { smoking history }\end{array}$ & $\begin{array}{l}\text { Predict age and sex } \\
\text { from retinal fundus } \\
\text { images }\end{array}$ & CNN & AUC 0.96 & Ophthalmology & $\begin{array}{l}{[2] ;} \\
{[158]}\end{array}$ \\
\hline & $\begin{array}{l}\text { Dermoscopy } \\
\text { images }\end{array}$ & $\begin{array}{l}350 \text { images of } \\
\text { melanomas and } \\
374 \text { benign nevus }\end{array}$ & $\begin{array}{l}\text { Dermoscopy CAD } \\
\text { system for acral } \\
\text { lentiginous melanoma } \\
\text { diagnosis }\end{array}$ & $\mathrm{CNN}$ & $\begin{array}{l}\text { Accuracy of over } \\
0.80\end{array}$ & Oncology & $\begin{array}{l}{[2] ;} \\
{[99]}\end{array}$ \\
\hline \multirow[t]{2}{*}{ Dermoscopy } & $\begin{array}{l}\text { Patient } \\
\text { demographics } \\
\text { and clinical } \\
\text { images }\end{array}$ & 49,567 images & $\begin{array}{l}\text { Recognize nails } \\
\text { nychomycosis lesions }\end{array}$ & $\begin{array}{l}\text { Region-based- } \\
\text { CNN }\end{array}$ & $\begin{array}{l}\text { AUC of } 0.98, \text { AUC } \\
\text { of } 0.95 \text {, AUC of } \\
0.93, \text { AUC value } \\
\text { of } 0.82 \text { in the } \\
\text { different } \\
\text { datasets }\end{array}$ & Dermatology & [120] \\
\hline & $\begin{array}{l}\text { Stress } \\
99 \mathrm{mTc} \text {-sestamibi } \\
\text { or tetrofosmin } \\
\text { myocardial } \\
\text { perfusion } \\
\text { images }\end{array}$ & 1638 patients & $\begin{array}{l}\text { Obstructive coronary } \\
\text { disease automatic } \\
\text { prediction system }\end{array}$ & $\mathrm{CNN}$ & $\begin{array}{l}\text { Sensitivity value of } \\
0.82 \text { and } 0.69 \text { for } \\
\text { both use cases }\end{array}$ & Cardiovascular & [159] \\
\hline Arterial labeling & $\begin{array}{l}\text { Arterial spin } \\
\text { labeling (ASL) } \\
\text { perfusion images }\end{array}$ & 140 subjects & $\begin{array}{l}\text { Monitoring cerebral } \\
\text { arterial perfusion via } \\
\text { spin labeling }\end{array}$ & $\mathrm{CNN}$ & AUC of 0.94 & Cardiovascular & [91] \\
\hline $\begin{array}{l}\text { Frames from } \\
\text { endoscopy }\end{array}$ & $\begin{array}{l}\text { Frames from } \\
\text { endoscopy videos }\end{array}$ & $\begin{array}{l}205 \text { normal and } \\
360 \text { abnormal } \\
\text { images }\end{array}$ & $\begin{array}{l}\text { Detection and } \\
\text { localization system } \\
\text { of gastrointestinal } \\
\text { anomalies via } \\
\text { endoscopy }\end{array}$ & CNN & AUC of over 0.80 & Gastroenterology & [103] \\
\hline
\end{tabular}


Continued

\begin{tabular}{|c|c|c|c|c|c|c|c|}
\hline \multirow[t]{4}{*}{$\begin{array}{l}\text { Tracking } \\
\text { dataset } \\
\text { multi- } \\
\text { instrument } \\
\text { Endo-Visceral } \\
\text { Surgery and } \\
\text { multi- } \\
\text { instrument in } \\
\text { vivo }\end{array}$} & $\begin{array}{l}\text { Single- } \\
\text { instrument } \\
\text { Retinal } \\
\text { Microsurgery } \\
\text { Instrument } \\
\text { Tracking dataset, } \\
\text { Multi-instrument } \\
\text { Endo-Visceral } \\
\text { surgery and } \\
\text { multi-instrument } \\
\text { in vivo images }\end{array}$ & $\begin{array}{l}940 \text { frames of the } \\
\text { training data ( } 4479 \\
\text { frames) and } 910 \\
\text { frames for the test } \\
\text { data ( } 4495 \text { frames) }\end{array}$ & $\begin{array}{l}\text { Detect the } \\
\text { two-dimensional } \\
\text { position of different } \\
\text { medical instruments } \\
\text { in endoscopy and } \\
\text { microscopy surge }\end{array}$ & $\begin{array}{l}\text { Convolutional } \\
\text { Detection } \\
\text { regression } \\
\text { network }\end{array}$ & Accuracy of 0.94 & Robotic Surgery & $\begin{array}{l}{[2]:} \\
{[119]}\end{array}$ \\
\hline & Nuclear MRIs 3D & $\begin{array}{l}124 \text { double echo } \\
\text { steady state from } \\
17 \text { patients }\end{array}$ & $\begin{array}{l}\text { Diagnose possible soft } \\
\text { tissue injuries }\end{array}$ & $\begin{array}{l}\text { DeepResolve, a } \\
\text { 3D-CNN model }\end{array}$ & MSE of 0.008 & Traumatology & $\begin{array}{l}{[2]} \\
{[160]}\end{array}$ \\
\hline & $\begin{array}{l}\text { Retinal 3D } \\
\text { images obtained } \\
\text { by Optical } \\
\text { Coherence } \\
\text { Tomography }\end{array}$ & $\begin{array}{l}269 \text { patients with } \\
\text { AMD, } 115 \text { control } \\
\text { patients }\end{array}$ & $\begin{array}{l}\text { Retina age-related } \\
\text { macular degeneration } \\
\text { diagnostic }\end{array}$ & $\mathrm{CNN}$ & AUC of 0 & Ophthalmology & [77] \\
\hline & $\begin{array}{l}\text { 123I-fluoropropyl } \\
\text { carbomethoxy- } \\
\text { iodophenyl } \\
\text { nortropane } \\
\text { single-photon } \\
\text { emission } \\
\text { computed } \\
\text { tomography } \\
\text { (FP-CIT SPECT) }\end{array}$ & 431 patient cases & $\begin{array}{l}\text { Automatic } \\
\text { interpretation system } \\
\text { in Parkinson's disease }\end{array}$ & $\mathrm{CNN}$ & Accuracy of 0.96 & $\begin{array}{l}\text { Neurology- } \\
\text { Psychiatry }\end{array}$ & [79] \\
\hline \multirow{4}{*}{$\begin{array}{l}\text { CT/PET- } \\
\text { CT/SPECT }\end{array}$} & 2D images & & & & & & \\
\hline & $\begin{array}{l}\text { Abdominal CT } \\
\text { 3D images }\end{array}$ & $\begin{array}{l}231 \text { computed } \\
\text { abdominal }\end{array}$ & $\begin{array}{l}\text { CAD system to } \\
\text { classify tomographyes } \\
\text { and evaluate the } \\
\text { malignity degree in } \\
\text { gastro-intestinal } \\
\text { stromal tumors } \\
\text { (GISTs) }\end{array}$ & $\begin{array}{l}\text { Hybrid system } \\
\text { between } \\
\text { convolutional } \\
\text { networks and } \\
\text { radiomics }\end{array}$ & AUC of 0.882 & Oncology & {$[161]$} \\
\hline & $\begin{array}{l}\text { CT image patches } \\
2 \mathrm{D}\end{array}$ & $\begin{array}{l}14,696 \text { images } \\
\text { from } 120 \text { patients } \\
\text { with proven } \\
\text { diagnose }\end{array}$ & $\begin{array}{l}\text { CAD system to } \\
\text { diagnose interstitial } \\
\text { lung disease }\end{array}$ & $\mathrm{CNN}$ & Accuracy of 0.85 & Pneumology & [85] \\
\hline & 3D MRI and PET & $\begin{array}{l}93 \text { Alzheimer } \\
\text { Disease, } 204 \text { MCI } \\
\text { Mild Cognitive } \\
\text { Impairment } \\
\text { converters and } \\
\text { normal control } \\
\text { subjects }\end{array}$ & $\begin{array}{l}\text { CAD for early } \\
\text { Alzheimer disease } \\
\text { stages }\end{array}$ & Multimodal DBM & $\begin{array}{l}\text { Accuracy of } 0.95 \text {, } \\
0.85 \text { and } 0.75 \text { for } \\
\text { the three use cases }\end{array}$ & $\begin{array}{l}\text { Neurology- } \\
\text { Psychiatry }\end{array}$ & $\begin{array}{l}{[2] ;} \\
{[92]}\end{array}$ \\
\hline $\begin{array}{l}\text { CT/PET- } \\
\text { CT/SPECT }\end{array}$ & $\begin{array}{l}\text { CT images, MRI } \\
\text { images and PET } \\
\text { images }\end{array}$ & $\begin{array}{l}6776 \text { images for } \\
\text { training and } 4166 \\
\text { for tests }\end{array}$ & $\begin{array}{l}\text { Classify medical } \\
\text { diagnostic images } \\
\text { according to the } \\
\text { modality they were } \\
\text { produced and classify } \\
\text { illustrations according } \\
\text { to their production } \\
\text { attributes }\end{array}$ & $\begin{array}{l}\mathrm{CNN} \text { and a } \\
\text { synergic signal } \\
\text { system }\end{array}$ & Accuracy of 0.86 & Various & $\begin{array}{l}{[2]} \\
{[108]}\end{array}$ \\
\hline
\end{tabular}




\begin{tabular}{|c|c|c|c|c|c|c|c|}
\hline & CT image 2D & $\begin{array}{l}63,890 \text { patients } \\
\text { with cancer and } \\
171,345 \text { healthy }\end{array}$ & $\begin{array}{l}\text { Discriminate lung } \\
\text { cancer lesions in } \\
\text { adenocarcinoma, } \\
\text { squamous and small } \\
\text { cell carcinoma }\end{array}$ & $\mathrm{CNN}$ & $\begin{array}{l}\text { Log-Loss error } \\
\text { of } 0.66 \text { with a } \\
\text { sensitivity of } 0.87\end{array}$ & Oncology & [118] \\
\hline & CT 2D images & $\begin{array}{l}3059 \text { images from } \\
\text { several parts of } \\
\text { human body }\end{array}$ & $\begin{array}{l}\text { Speed up CT images } \\
\text { collection and rebuild } \\
\text { the data }\end{array}$ & $\begin{array}{l}\text { Dense Net and a } \\
\text { deconvolution } \\
\text { model }\end{array}$ & RMSE of 0.00048 & Various & {$[11]$} \\
\hline & CT images 3D & $\begin{array}{l}6960 \text { lung nodule } \\
\text { regions, } 3480 \text { of } \\
\text { which were } \\
\text { positive samples } \\
\text { and rest were } \\
\text { negative samples } \\
\text { (nonnodule) }\end{array}$ & $\begin{array}{l}\text { CAD to diagnose lung } \\
\text { cancer in low-dosage } \\
\text { computed tomography }\end{array}$ & $\begin{array}{l}\text { Eye tracking } \\
\text { sparse attentional } \\
\text { model and } \\
\text { convolutional } \\
\text { neural network }\end{array}$ & Accuracy of 0.97 & Oncology & [162] \\
\hline & $\begin{array}{l}\text { CT images 2D } \\
\text { and text (reports) }\end{array}$ & $\begin{array}{l}9000 \text { training and } \\
1000 \text { testing } \\
\text { images }\end{array}$ & $\begin{array}{l}\text { Processing text from } \\
\text { CT reports in order to } \\
\text { classify their respective } \\
\text { images }\end{array}$ & $\mathrm{CNN}$ & $\begin{array}{l}\text { Accuracy of } 0.95 \text {, } \\
0.70 \text { and } 0.58 \\
\text { respectively for the } \\
\text { three use cases }\end{array}$ & Various & [12] \\
\hline & $\begin{array}{l}\text { Computed } \\
\text { tomography (CT) }\end{array}$ & $\begin{array}{l}\text { Three datasets: } \\
224,316,112,120 \\
\text { and } 15,783\end{array}$ & $\begin{array}{l}\text { Binary classification of } \\
\text { posteroanterior chest } \\
\text { xray }\end{array}$ & CNN & $92 \%$ accuracy & Radiology & $\begin{array}{l}{[2]} \\
{[163]}\end{array}$ \\
\hline & $\begin{array}{l}\text { Diffusion- } \\
\text { weighted imaging } \\
\text { maps using MRI }\end{array}$ & $\begin{array}{l}222 \text { patients. } 187 \\
\text { treated with rtPA } \\
\text { (recombinant } \\
\text { tissue-type } \\
\text { plasminogen } \\
\text { activator) }\end{array}$ & $\begin{array}{l}\text { Decide Acute Ischemic } \\
\text { Stroke patients' } \\
\text { treatment through } \\
\text { volume lesions } \\
\text { prediction }\end{array}$ & $\mathrm{CNN}$ & AUC of 0.88 & $\begin{array}{l}\text { Neurology- } \\
\text { Psychiatry }\end{array}$ & $\begin{array}{l}{[2] ;} \\
{[81]}\end{array}$ \\
\hline & $\begin{array}{l}\text { Magnetic } \\
\text { resonance } \\
\text { images }\end{array}$ & $\begin{array}{l}474 \text { patients with } \\
\text { schizophrenia and } \\
607 \text { healthy } \\
\text { subjects }\end{array}$ & $\begin{array}{l}\text { Schizophrenia } \\
\text { detection }\end{array}$ & $\begin{array}{l}\text { Deep discriminant } \\
\text { autoencoder } \\
\text { network }\end{array}$ & Accuracy over 0.8 & $\begin{array}{l}\text { Neurology- } \\
\text { Psychiatry }\end{array}$ & [83] \\
\hline & $\begin{array}{l}\text { Gadoxetic } \\
\text { acid-enhanced 2D } \\
\text { MRI }\end{array}$ & $\begin{array}{l}144,180 \text { images } \\
\text { from } 634 \text { patients }\end{array}$ & $\begin{array}{l}\text { Staging liver fibrosis } \\
\text { through MR }\end{array}$ & $\mathrm{CNN}$ & $\begin{array}{l}\text { AUC values of } \\
0.84,0.84 \text {, and } 0.85 \\
\text { for each stage }\end{array}$ & Gastroenterology & [86] \\
\hline \multirow[t]{2}{*}{ MRI } & $\begin{array}{l}\text { Resting state } \\
\text { functional } \\
\text { magnetic } \\
\text { resonance } \\
\text { imaging } \\
\text { (rs-fMRI), T1 } \\
\text { structural cerebra } \\
\text { images and } \\
\text { phenotypic } \\
\text { information }\end{array}$ & $\begin{array}{l}505 \text { individuals } \\
\text { with autism and } \\
520 \text { matched } \\
\text { ltypical controls }\end{array}$ & $\begin{array}{l}\text { Identify different } \\
\text { autism spectrum } \\
\text { disorders }\end{array}$ & Denoising AE & Accuracy of 0.70 & $\begin{array}{l}\text { Neurology- } \\
\text { Psychiatry }\end{array}$ & [92] \\
\hline & 3D MRI and PET & $\begin{array}{l}93 \text { Alzheimer } \\
\text { Disease, } 204 \text { MCI } \\
\text { Mild Cognitive } \\
\text { Impairment } \\
\text { converters and } \\
\text { normal control } \\
\text { subjects }\end{array}$ & $\begin{array}{l}\text { CAD for early } \\
\text { Alzheimer disease } \\
\text { stages }\end{array}$ & Multimodal DBM & $\begin{array}{l}\text { Accuracy of } 0.95 \text {, } \\
0.85 \text { and } 0.75 \text { for } \\
\text { the three use cases }\end{array}$ & $\begin{array}{l}\text { Neurology- } \\
\text { Psychiatry }\end{array}$ & $\begin{array}{l}{[2] ;} \\
{[93]}\end{array}$ \\
\hline
\end{tabular}


Lung computed axial tomography 2D images and breast ultrasound lesions

MRI 2D

MRI 2D

CT images, MRI images and PET 6776 images images

MRI

Liver MRIs

patients $(700$

68 subjects

Functional MRI

perform 7

state of rest
135 patients with Predict the survival

short-medium and of patients with amyotrophic lateral

CNN

long-term survival sclerosis

Differentiate

Age-Related Macular

atients' images Degeneration lesions in and 48,312 control optical coherence

tomography

Modification of

Modification of

520 breast

sonograms from

520 patients $(275$

benign and 245 CAD system to classify

malignant lesions) breast ultrasound

and lung CT image lesions and lung CT AE

Stacked denoising $\mathrm{AE}$

AUC of $0.94 \quad$ Oncology

[164]

data from 1010 nodules

malignant and 700

benign nodules)

444 images from

195 patients with prostate cancer

CAD to prevent errors

in diagnosing prostate

$\mathrm{CNN}$

AUC of $0.94 \quad$ Oncology

[165]

MICCAI 2009 left Determinate limits

ventricle between the

endocardium and

segmentation epicardium of the left challenge database ventricle

RNN with

automatic

segmentation

techniques

Classify medical

diagnostic images

according to the

modality they were

produced and classify

illustrations according

CNN and a

synergic signal system

Accuracy of 0.86 Various

to their production

attributes

activities, and a

Analyze cerebral

cognitive functions

3D CNN, resting

state networks

Accuracy of 0.94

Neurology-

522 liver MRI cases

with and without Screening system for contrast for known undiagnosed hepatic or suspected liver magnetic resonance

$\mathrm{CNN}$

Reduces negative predictive value and leads to

Gastroenterology greater precision

liver lesion

1064 brain images

of autism patients

and healthy

Automatically

controls. MRI

evaluate the quality of

multicenter structural

$\mathrm{CNN}$

AUC 0.90 and 0.71 Radiology

brain MRI images

multiple sclerosis

patient 


\section{Conclusions}

Doctors interpret images descriptively (contour, contrast, appearance, localization, etc.) by using data from different excipients and successive stages in the analysis of medical images. These handcrafted features consume time and do not have a standardized character.

Data quality and volume, annotations and labels, identification and automatic extraction of specific medical terms can help deep learning models perform in the tasks of image analysis [3]. Incorporating these features, labels, into DL architectures increases their performance.

High-level domain knowledge is incorporated as input images [3], and low-level domain knowledge is learned using specific network structures [35] and, together with direct networking, low-level domain knowledge information can also be used to design training commands when combined with the easy-to-use training model [3] [133].

DL can be a support in solving complex problems of interpretation of medical images and provides the doctor with support in making medical decisions and time for patient care.

\section{Research Problems}

Problems in medical image analysis can be categorized as follows:

- identification and automatic extraction and standardization of specific medical terms,

- representation of medical knowledge,

- incorporation of medical knowledge.

Problems in medical image analysis are related to:

- medical images provided as data for deep-street models require: quality, volume, specificity, labelling.

- providing data from doctors, descriptive data, labels are ambiguous for the same medical and non-standard references.

- laborious time in data processing are problems to solve in the future.

- lack of clinical trials demonstrating the benefits of using DL medical applications in reducing morbidity and mortality and improving patient quality of life [4].

\section{Future Challenges}

These consist of adapting the domain consisting of transferring data from one domain to another domain by using labels; knowledge graph characterized by the incorporation of multimodal medical data; generating models capable of extracting features unsupervised and easily incorporated into the architecture of DL networks; techniques to search for a particular network architecture according to the defined objectives.

The adaptation of the domain consisted of transferring information from a source domain to a target domain [3], such as adversarial learning [134], and 
makes it restrict the domain change between source and target [3] domain in input space [135], feature space [136] [137] and output space [138] [139]. It can be used to transfer knowledge about one set of medical data to another [3] [140], even when they have different modes [3] of imaging or belong to different diseases [141] [142]. UDA (unsupervised domain adaptation), which uses medical labels, has demonstrated performance in disease diagnosis and organ segmentation [3] [140] [143] [144] [145].

The knowledge graph has the specifics of incorporating multimodal medical data and achieves performance in medical image analysis [3] and the creation of medical reports [146]. The graphs of medical knowledge describing, the relationship between different types of knowledge, the relationship between different diseases, the relationship between medical datasets and a type of medical data, help deep learning models work [147].

Generating models, GAN and AE are mainly used for segmentation activities. GAN uses MRI datasets to segment CT images [142] [143]. GAN is a type of unsupervised deep learning network used in medical image analysis [3] [167]. AE are used in extracting features, shape priorities in objects such as organs or lesions, completely unsupervised and are easily incorporated into the network formation process [35] [148].

Network Architecture Search Technique (NAS) can automatically identify a specific network architecture in computer tasks [149] and promises that utility and performance in the medical field [150].

\section{Funding}

Scientific research funded by the University of Medicine and Pharmacy "Gr. T. Popa" of Iasi, based on contract number 4714 .

\section{Conflicts of Interest}

The authors declare no conflicts of interest regarding the publication of this paper.

\section{References}

[1] Pandey, B., Pandey, D.K., Mishra, B.P. and Rhmann, W. (2021) A Comprehensive Survey of Deep Learning in the Field of Medical Imaging and Medical Natural Language Processing: Challenges and Research Directions. Journal of King Saud University - Computer and Information Sciences, in press. https://doi.org/10.1016/j.jksuci.2021.01.007

[2] Nogales, A., García-Tejedor, Á.J., Monge, D., Vara, J.S. and Antón, C. (2021) A Survey of Deep Learning Models in Medical Therapeutic Areas. Artificial Intelligence in Medicine, 112, Article ID: 102020. https://doi.org/10.1016/j.artmed.2021.102020

[3] Xie, X.Z., Niu, J.W., Liu, X.F., Chen, Z.S., Tang, S.J. and Yu, S. (2021) A Survey on Incorporating Domain Knowledge into Deep Learning for Medical Image Analysis. Medical Image Analysis, 69, Article ID: 101985.

https://doi.org/10.1016/j.media.2021.101985 
[4] Piccialli, F., Di Somma, V., Giampaolo, F., Cuomo, S. and Fortino, G. (2021) A Survey on Deep Learning in Medicine: Why, How and When? Information Fusion, 66, 111137. https://doi.org/10.1016/j.inffus.2020.09.006

[5] Shin, H.-C., Lu, L. and Summers, R.M. (2017) Chapter 17. Natural Language Processing for Large-Scale Medical Image Analysis Using Deep Learning. In: Zhou, S.K., Greenspan, H. and Shen, D.G., Eds., Deep Learning for Medical Image Analysis, Academic Press, Cambridge, MA, 405-421. https://doi.org/10.1016/B978-0-12-810408-8.00023-7

[6] Wang, X., Yang, X., Dou, H.R., Li, S.L., Heng, P. and Ni, D. (2019) Joint Segmentation and Landmark Localization of Fetal Femur in Ultrasound Volumes. IEEE EMBS International Conference on Biomedical \& Health Informatics (BHI), Chicago, IL, 19-22 May 2019, 1-5. https://doi.org/10.1109/BHI.2019.8834615

[7] Sharma, S. and Mehra, R. (2020) Conventional Machine Learning and Deep Learning Approach for Multi-Classification of Breast Cancer Histopathology Images-A Comparative Insight. Journal of Digital Imaging, 33, 632-654. https://doi.org/10.1007/s10278-019-00307-y

[8] Pattanaik, P.A., Mittal, M., Khan, M.Z. and Panda, S.N. (2020) Malaria Detection Using Deep Residual Networks with Mobile Microscopy. Journal of King Saud University-Computer and Information Sciences, in Press.

https://doi.org/10.1016/j.jksuci.2020.07.003

[9] He, Y.T., Yang, G.Y., Chen, Y., Kong, Y.Y., Wu, J.S., et al. (2019) DPA-DenseBiasNet: Semi-Supervised 3D Fine Renal Artery Segmentation with Dense Biased Network and Deep Priori Anatomy. In: Shen, D., et al., Eds., Medical Image Computing and Computer Assisted Intervention-MICCAI 2019. MICCAI 2019. Lecture Notes in Computer Science, Vol. 11769, Springer, Cham, 139-147.

https://doi.org/10.1007/978-3-030-32226-7_16

[10] Zhu, Y., Wang, M.D., Tong, L. and Deshpande, S.R. (2019) Improved Prediction on Heart Transplant Rejection Using Convolutional Autoencoder and Multiple Instance Learning on Whole-Slide Imaging. IEEE EMBS International Conference on Biomedical and Health Informatics, Chicago, IL, 19-22 May 2019, 1-4. https://doi.org/10.1109/BHI.2019.8834632

[11] Zhang, Z., Liang, X., Dong, X., Xie, Y. and Cao, G. (2018) A Sparse-View CT Reconstruction Method Based on Combination of DenseNet and Deconvolution. IEEE Transactions on Medical Imaging, 37, 1407-1417. https://doi.org/10.1109/TMI.2018.2823338

[12] Shin, H.-C., Lu, L., Kim, L., Seff, A., Yao, J.H. and Summers, R.M. (2016) Interleaved Text/Image Deep Mining on a Large-Scale Radiology Database for Automated Image Interpretation. Journal of Machine Learning Research, 17, 1-31.

[13] Ravi, D., Wong, C., Deligianni, F., Berthelot, M., Andreu-Perez, J., Lo, B. and Yang, G.Z. (2017) Deep Learning for Health Informatics. IEEE Journal of Biomedical and Health Informatics, 21, 4-21. https://doi.org/10.1109/JBHI.2016.2636665

[14] Vizcarra, J., Place, R., Tong, L., Gutman, D. and Wang, M.D. (2019) Fusion in Breast Cancer Histology Classification. Proceedings of the 10 th ACM International Conference on Bioinformatics, Computational Biology and Health Informatics, Niagara Falls, NY, 7-10 September 2019, 485-493. https://doi.org/10.1145/3307339.3342166

[15] Zhao, Y., Dong, Q., Zhang, S., Zhang, W., Chen, H., Jiang, X., Guo, L., Hu, X., Han, J. and Liu, T. (2018) Automatic Recognition of fMRI-Derived Functional Networks Using 3-D Convolutional Neural Networks. IEEE Transactions on Biomedical Engineering, 65, pp1975-1984. https://doi.org/10.1109/TBME.2017.2715281 
[16] Esteva, A., Kuprel, B., Novoa, R.A., Ko, J., Swetter, S.M., Blau, H.M. and Thrun, S. (2017) Dermatologist-Level Classification of Skin Cancer with Deep Neural Networks. Nature. Nature, 542, 115-118. https://doi.org/10.1038/nature21056

[17] Zhou, H., Sun, J., Yacoob, Y. and Jacobs, D.W. (2018) Label Denoising Adversarial Network (LDAN) for Inverse Lighting of Faces. 2018 IEEE/CVF Conference on Computer Vision and Pattern Recognition, Salt Lake City, 18-23 June 2018, 62386247. https://doi.org/10.1109/CVPR.2018.00653

[18] Zhu, W.T., Liu, C.C., Fan, W. and Xie, X. (2018) DeepLung: Deep 3D Dual Path Nets for Automated Pulmonary Nodule Detection and Classification. 2018 IEEE Winter Conference on Applications of Computer Vision (WACV), Lake Tahoe, 12-15 March 2018, 673-681. https://doi.org/10.1109/WACV.2018.00079

[19] Zhang, R., Zheng, Y., Poon, C.C.Y., Shen, D. and Lau, J.Y.W. (2018) Polyp Detection during Colonoscopy Using a Regression-Based Convolutional Neural Network with a Tracker. Pattern Recognition, 83, 209-219.

https://doi.org/10.1016/j.patcog.2018.05.026

[20] Cheimariotis, G.A., Riga, M., Toutouzas, K., Tousoulis, D., Katsaggelos, A. and Maglaveras, N. (2019) Deep Learning Method to Detect Plaques in IVOCT Images. In: Lin, K.-P., Magjarevic, R. and de Carvalho, P., Eds., Future Trends in Biomedical and Health Informatics and Cybersecurity in Medical Devices. ICBHI 2019. IFMBE Proceedings, Vol. 74, Springer, Cham, 389-395. https://doi.org/10.1007/978-3-030-30636-6 53

[21] Halevy, A., Norvig, P. and Pereira, F. (2009) The Unreasonable Effectiveness of Data. IEEE Intelligent Systems, 24, 8-12. https://doi.org/10.1109/MIS.2009.36

[22] Shin, H.C., Roth, H.R., Gao, M., Lu, L., Xu, Z., Nogues, I., et al. (2016) Deep Convolutional Neural Networks for Computer-Aided Detection: CNN Architectures, Dataset Characteristics and Transfer Learning. IEEE Transactions on Medical Imaging, 35, 1285-1298. https://doi.org/10.1109/TMI.2016.2528162

[23] Tajbakhsh, N., Shin, J.Y., Gurudu, S.R., Hurst, R.T., Kendall, C.B., Gotway, M.B. and Liang, J.M. (2016) Convolutional Neural Networks for Medical Image Analysis: Full Training or Fine Tuning. IEEE Transactions on Medical Imaging, 35, 12991312. https://doi.org/10.1109/TMI.2016.2535302

[24] Yap, M.H., Pons, G., Marti, J., Ganau, S., Sentis, M., Zwiggelaar, R., Davison, A.K., Marti, R., Yap, M.H., Pons, G., Marti, J., Ganau, S., Sentis, M., Zwiggelaar, R., Davison, A.K. and Marti, R. (2018) Automated Breast Ultrasound Lesions Detection Using Convolutional Neural Networks. IEEE Journal of Biomedical and Health Informatics, 22, 1218-1226. https://doi.org/10.1109/JBHI.2017.2731873

[25] Näppi, J.J., Hironaka, T., Regge, D. and Yoshida, H. (2016) Deep Transfer Learning of Virtual Endoluminal Views for the Detection of Polyps in CT Colonography. Medical Imaging 2016: Computer-Aided Diagnosis, 9785, 97852B. https://doi.org/10.1117/12.2217260

[26] Zhang, R., Zheng, Y., Mak, T.W., Yu, R., Wong, S.H., Lau, J.Y. and Poon, C.C. (2017) Automatic Detection and Classification of Colorectal Polyps by Transferring LowLevel CNN Features From Nonmedical Domain. IEEE Journal of Biomedical and Health Informatics, 21, 41-47. https://doi.org/10.1109/JBHI.2016.2635662

[27] Tran, D., Bourdev, L., Fergus, R., Torresani, L. and Paluri, M. (2015) Learning Spatiotemporal Features with 3D Convolutional Networks. IEEE International Conference on Computer Vision (ICCV), Santiago, 7-13 December 2015, 4489-4497. https://doi.org/10.1109/ICCV.2015.510

[28] Samala, R.K., Chan, H.P., Hadjiiski, L.M., Helvie, M.A., Cha, K.H. and Richter, C.D. 
(2017) Multi-Task Transfer Learning Deep Convolutional Neural Network: Application to Computer-Aided Diagnosis of Breast Cancer on Mammograms. Physics in Medicine \& Biology, 62, 8894-8908. https://doi.org/10.1088/1361-6560/aa93d4

[29] Samala, R.K., Chan, H.-P., Hadjiiski, L., Helvie, M.A., Richter, C.D. and Cha, K.H. (2019) Breast Cancer Diagnosis in Digital Breast Tomosynthesis: Effects of Training Sample Size on Multi-Stage Transfer Learning Using Deep Neural Nets. IEEE Transactions on Medical Imaging, 38, 686-696.

https://doi.org/10.1109/TMI.2018.2870343

[30] Liao, Q., Ding, Y., Jiang, Z.L., Wang, X., Zhang, C.K. and Zhang, Q. (2019) MultiTask Deep Convolutional Neural Network for Cancer Diagnosis. Neurocomputing, 348, 66-73. https://doi.org/10.1016/j.neucom.2018.06.084

[31] Ben-Cohen, A., Klang, E., Raskin, S.P., Soffer, S., Ben-Haim, S., Konen, E., Amitai, M.M. and Greenspan, H. (2019) Cross-Modality Synthesis from CT to PET Using FCN and GAN Networks for Improved Automated Lesion Detection. Engineering Applications of Artificial Intelligence, 78, 186-194. https://doi.org/10.1016/j.engappai.2018.11.013

[32] Zhao, J., Li, D., Kassam, Z., Howey, J., Chong, J., Chen, B. and Li, S. (2020) Tripartite-GAN: Synthesizing Liver Contrast-Enhanced MRI to Improve Tumor Detection. Medical Image Analysis, 63, Article ID: 101667. https://doi.org/10.1016/j.media.2020.101667

[33] Zhang, J., Saha, A., Zhu, Z. and Mazurowski, M.A. (2019) Hierarchical Convolutional Neural Networks for Segmentation of Breast Tumors in MRI with Application to Radiogenomics. IEEE Transactions on Medical Imaging, 38, 435-447. https://doi.org/10.1109/TMI.2018.2865671

[34] Yu, F., Zhao, J., Gong, Y., Wang, Z., Li, Y., Yang, F. and Zhang, L. (2019) Annotation-Free Cardiac Vessel Segmentation via Knowledge Transfer from Retinal Images. In: Shen, D., et al., Eds., Medical Image Computing and Computer Assisted Intervention-MICCAI 2019. MICCAI 2019. Lecture Notes in Computer Science, Vol. 11765, Springer, Cham, 714-722. https://doi.org/10.1007/978-3-030-32245-8 79

[35] Chen, C., Biffi, C., Tarroni, G., Petersen, S., Bai, W. and Rueckert, D. (2019) Learning Shape Priors for Robust Cardiac MR Segmentation from Multi-View Images. In: Shen, D., et al., Eds., Medical Image Computing and Computer Assisted Intervention-MICCAI 2019. MICCAI 2019. Lecture Notes in Computer Science, Vol. 11765, Springer, Cham, 523-531. https://doi.org/10.1007/978-3-030-32245-8 58

[36] Valindria, V.V., et al. (2018) Multi-Modal Learning from Unpaired Images: Application to Multi-Organ Segmentation in CT and MRI. 2018 IEEE Winter Conference on Applications of Computer Vision (WACV), Lake Tahoe, NV, 12-15 March 2018, 547-556. https://doi.org/10.1109/WACV.2018.00066

[37] Qin, C., Schlemper, J., Caballero, J., Price, A.N., Hajnal, J.V. and Rueckert, D. (2019) Convolutional Recurrent Neural Networks for Dynamic MR Image Reconstruction. IEEE Transactions on Medical Imaging, 38, 280-290. https://doi.org/10.1109/TMI.2018.2863670

[38] Schlemper, J., Caballero, J., Hajnal, J.V., Price, A.N. and Rueckert, D. (2018) A Deep Cascade of Convolutional Neural Networks for Dynamic MR Image Reconstruction. IEEE Transactions on Medical Imaging, 37, 491-503.

https://doi.org/10.1109/TMI.2017.2760978

[39] Yang, G., Yu, S., Dong, H., Slabaugh, G., Dragotti, P.L., Ye, X., Liu, F., Arridge, S., Keegan, J., Guo, Y., Firmin, D., Keegan, J., Slabaugh, G., Arridge, S., Ye, X., Guo, Y., Yu, S., Liu, F., Firmin, D., Dragotti, P.L., Yang, G. and Dong, H. (2018) DAGAN: 
Deep De-Aliasing Generative Adversarial Networks for Fast Compressed Sensing MRI Reconstruction. IEEE Transactions on Medical Imaging, 37, 1310-1321. https://doi.org/10.1109/TMI.2017.2785879

[40] Ben Yedder, H., Shokoufi, M., Cardoen, B., Golnaraghi, F. and Hamarneh, G. (2019) Limited-Angle Diffuse Optical Tomography Image Reconstruction Using Deep Learning. In: Shen, D., et al., Eds., Medical Image Computing and Computer Assisted Intervention-MICCAI 2019. MICCAI 2019. Lecture Notes in Computer Science, Vol. 11764, Springer, Cham, 66-74. https://doi.org/10.1007/978-3-030-32239-7 8

[41] Ahmad, J., Sajjad, M., Mehmood, I. and Baik, S.W. (2017) SiNC: Saliency-Injected Neural Codes for Representation and Efficient Retrieval of Medical Radiographs. PLoS ONE, 12, e0181707. https://doi.org/10.1371/journal.pone.0181707

[42] Khatami, A., Babaie, M., Tizhoosh, H.R., Khosravi, A., Nguyen, T. and Nahavandi, S. (2018) A Sequential Search-Space Shrinking Using CNN Transfer Learning and a Radon Projection Pool for Medical Image Retrieval. Expert Systems with Applications, 100, 224-233. https://doi.org/10.1016/j.eswa.2018.01.056

[43] Swati, Z.N.K., Zhao, Q., Kabir, M., Ali, F., Ali, Z., Ahmed, S. and Lu, J. (2019) Content-Based Brain Tumor Retrieval for MR Images Using Transfer Learning. IEEE Access, 7, 17809-17822. https://doi.org/10.1109/ACCESS.2019.2892455

[44] Pham, H.H., Le, T.T., Tran, D.Q., Ngo, D.T. and Nguyen, H.Q. (2019) Interpreting Chest X-Rays via CNNs That Exploit Hierarchical Disease Dependencies and Uncertainty Labels. arXiv: 1911.06475 https://doi.org/10.1101/19013342

[45] Bekker, A.J. and Goldberger, J. (2016) Training Deep Neural-Networks Based on Unreliable Labels. 2016 IEEE International Conference on Acoustics, Speech and Signal Processing (ICASSP), Shanghai, 20-25 March 2016, 2682-2686.

https://doi.org/10.1109/ICASSP.2016.7472164

[46] Matuszewski, D.J. and Sintorn, I.M. (2018) Minimal Annotation Training for Segmentation of Microscopy Images. 2018 IEEE 15th International Symposium on Biomedical Imaging (ISBI 2018), Washington DC, 4-7 April 2018, 387-390. https://doi.org/10.1109/ISBI.2018.8363599

[47] Ren, M., Zeng, W., Yang, B. and Urtasun, R. (2018) Learning to Reweight Examples for Robust Deep Learning. Proceedings of the 35th International Conference on Machine Learning, PMLR, 80, 4334-4343.

[48] Xue, C., Dou, Q., Shi, X., Chen, H. and Heng, P.A. (2019) Robust Learning at Noisy Labeled Medical Images: Applied to Skin Lesion Classification. 2019 IEEE 16th International Symposium on Biomedical Imaging (ISBI 2019), Venice, 8-11 April 2019, 1280-1283. https://doi.org/10.1109/ISBI.2019.8759203

[49] Mirikharaji, Z., Yan, Y. and Hamarneh, G. (2019) Learning to Segment Skin Lesions from Noisy Annotations. In: Wang, Q., et al., Eds., Domain Adaptation and Representation Transfer and Medical Image Learning with Less Labels and Imperfect Data. DART 2019, MIL3ID 2019. Lecture Notes in Computer Science, Vol. 11795, Springer, Cham, 207-215. https://doi.org/10.1007/978-3-030-33391-1 24

[50] Nie, D., Gao, Y., Wang, L. and Shen, D. (2018) ASDNet: Attention Based SemiSupervised Deep Networks for Medical Image Segmentation. In: Frangi, A., Schnabel, J., Davatzikos, C., Alberola-López, C. and Fichtinger, G., Eds., Medical Image Computing and Computer Assisted Intervention-MICCAI 2018. MICCAI 2018. Lecture Notes in Computer Science, Vol. 11073, Springer, Cham, 370-378. https://doi.org/10.1007/978-3-030-00937-3 43

[51] Fries, J.A., Varma, P., Chen, V.S., Xiao, K., Tejeda, H., Saha, P., Dunnmon, J., Chubb, H., Maskatia, S., Fiterau, M., Delp, S., Ashley, E., Ré, C. and Priest, J.R. (2019) 
Weakly Supervised Classification of Aortic Valve Malformations Using Unlabeled Cardiac MRI Sequences. Nature Communications, 10, Article No. 3111. https://doi.org/10.1038/s41467-019-11012-3

[52] Jiang, F., Jiang, Y., Zhi, H., Dong, Y., Li, H., Ma, S., Wang, Y., Dong, Q., Shen, H. and Wang, Y. (2017) Artificial Intelligence in Healthcare: Past, Present and Future. Stroke and Vascular Neurology, 2, 230-243.

https://doi.org/10.1136/svn-2017-000101

[53] Miller, D.D. and Brown, E.W. (2018) Artificial Intelligence in Medical Practice: The Question to the Answer. The American Journal of Medicine, 131, 129-133. https://doi.org/10.1016/j.amjmed.2017.10.035

[54] Jang, H.J. and Cho, K.O. (2019) Applications of Deep Learning for the Analysis of Medical Data. Archives of Pharmacal Research, 42, 492-504.

https://doi.org/10.1007/s12272-019-01162-9

[55] Bakator, M. and Radosav, D. (2018) Deep Learning and Medical Diagnosis: A Review of Literature. Multimodal Technologies and Interaction, 2, 47. https://doi.org/10.3390/mti2030047

[56] Lundervold, A.S. and Lundervold, A. (2019) An Overview of Deep Learning in Medical Imaging Focusing on MRI. Zeitschrift für Medizinische Physik, 29, 102-127. https://doi.org/10.1016/j.zemedi.2018.11.002

[57] Hecht-Nielsen, R. (1988) Neurocomputing: Picking the Human Brain. IEEE Spectrum, 25, 36-41. https://doi.org/10.1109/6.4520

[58] Krizhevsky, A., Sutskever, I. and Hinton, G.E. (2012) ImageNet Classification with Deep Convolutional Neural Networks. In: Pereira, F., Burges, C.J.C., Bottou, L. and Weinberger, K.Q., Eds., Advances in Neural Information Processing Systems, Vol. 25, Curran Associates, Inc., Red Hook, NY, 1097-1105.

[59] Arasu, A. and Garcia-Molina, H. (2003) Extracting Structured Data from Web Pages. Proceedings of the 2003 ACM SIGMOD International Conference on Management of Data, San Diego, CA, 9-12 June 2003, 337-348. https://doi.org/10.1145/872757.872799

[60] Velicer, W.F. and Molenaar, P.C. (2012) Time Series Analysis for Psychological Research. In: Weiner, I., Schinka, J.A. and Velicer, W.F., Eds., Handbook of Psychology, 2nd Edition, John Wiley \& Sons, Inc, Hoboken. https://doi.org/10.1002/9781118133880.hop202022

[61] LeCun, Y., Boser, B., Denker, J.S., Henderson, D., Howard, R.E., Hubbard, W. and Jackel, L.D. (1989) Backpropagation Applied to Handwritten Zip Code Recognition. Neural Computation, 1, 541-551. https://doi.org/10.1162/neco.1989.1.4.541

[62] Krizhevsky, A., Sutskever, I. and Hinton, G.E. (2017) ImageNet Classification with Deep Convolutional Neural Networks. Communications of the ACM, 60, 84-90. https://doi.org/10.1145/3065386

[63] Kipf, T.N. and Welling, M. (2016) Semi-Supervised Classification with Graph Convolutional Networks. 5th International Conference on Learning Representations (ICLR-17). arXiv: 1609.02907.

[64] Elman, J.L. (1990) Finding Structure in Time. Cognitive Science, 14, 179-211. https://doi.org/10.1207/s15516709 $\operatorname{cog} 1402 \_1$

[65] Simonyan, K. and Zisserman, A. (2014) Very Deep Convolutional Networks for Large-Scale Image Recognition. arXiv: 1409.1556.

[66] León, J., Escobar, J.J., Ortiz, A., Ortega, J., González, J., Martín-Smith, P., Gan, J.Q. and Damas, M. (2020) Deep Learning for EEG-Based Motor Imagery Classification: 
Accuracy-Cost Trade-Off. PLoS ONE, 15, e0234178. https://doi.org/10.1371/journal.pone.0234178

[67] Hochreiter, S. and Schmidhuber, J. (1997) Long Short-Term Memory. Neural Computation, 9, 1735-1780. https://doi.org/10.1162/neco.1997.9.8.1735

[68] Sathya, R. and Abraham, A. (2013) Comparison of Supervised and Unsupervised Learning Algorithms for Pattern Classification. International Journal of Advanced Research in Artificial Intelligence (IJARAI), 2, 34-38. https://doi.org/10.14569/IJARAI.2013.020206

[69] Gondara, L. (2016) Medical Image Denoising Using Convolutional Denoising Autoencoders. 2016 IEEE 16th International Conference on Data Mining Workshops (ICDMW), Barcelona, 12-15 December 2016, 241-246. https://doi.org/10.1109/ICDMW.2016.0041

[70] Zhou, B.L., Khosla, A., Lapedriza, A., Torralba, A. and Oliva, A. (2016) Places: An Image Database for Deep Scene Understanding. arXiv: 1610.02055

[71] Nowling, R.J., et al. (2019) Classification before Segmentation: Improved U-Net Prostate Segmentation. 2019 IEEE EMBS International Conference on Biomedical \& Health Informatics (BHI), Chicago, IL, 19-22 May 2019, 1-4. https://doi.org/10.1109/BHI.2019.8834494

[72] Pesteie, M, Abolmaesumi, P. and Rohling, R.N. (2019) Adaptive Augmentation of Medical Data Using Independently Conditional Variational Auto-Encoders. IEEE Transactions on Medical Imaging, 38, 2807-2820. https://doi.org/10.1109/TMI.2019.2914656

[73] Yu, E.M., Iglesias, J.E., Dalca, A.V. and Sabuncu, M.R. (2020) An Auto-Encoder Strategy for Adaptive Image Segmentation. Proceedings of the Third Conference on Medical Imaging with Deep Learning, PMLR, 121, 881-891.

[74] Uzunova, H., Schultz, S., Handels, H., et al. (2019) Unsupervised Pathology Detection in Medical Images Using Conditional Variational Autoencoders. International Journal of Computer Assisted Radiology and Surgery 14, 451-461. https://doi.org/10.1007/s11548-018-1898-0

[75] Chen, M., Shi, X., Zhang, Y., Wu, D. and Guizani, M. (2017) Deep Features Learning for Medical Image Analysis with Convolutional Autoencoder Neural Network. IEEE Transactions on Big Data.

[76] Saltz, J., et al. (2018) Spatial Organization and Molecular Correlation of Tumor-Infiltrating Lymphocytes Using Deep Learning on Pathology Images. Cell Reports, 23, 181-193.e7.

[77] Apostolopoulos, S., Ciller, C., De Zanet, S., Wolf, S. and Sznitman, R. (2017) RetiNet: Automatic AMD Identification in OCT Volumetric Data. Investigative Ophthalmology \& Visual Science, 58, 387.

[78] Lam, C., Yu, C., Huang, L. and Rubin, D. (2018) Retinal Lesion Detection with Deep Learning Using Image Patches. Investigative Ophthalmology \& Visual Science, 59, 590-596. https://doi.org/10.1167/iovs.17-22721

[79] Choi, H., Ha, S., Im, H.J., Paek, S.H. and Lee, D.S. (2017) Refining Diagnosis of Parkinson's Disease with Deep Learning-Based Interpretation of Dopamine Transporter Imaging. NeuroImage: Clinical, 16, 586-594.

https://doi.org/10.1016/j.nicl.2017.09.010

[80] Rajaraman, S., Antani, S.K., Poostchi, M., Silamut, K., Hossain, M.A., Maude, R.J., Jaeger, S. and Thoma, G.R. (2018) Pre-Trained Convolutional Neural Networks as Feature Extractors toward Improved Malaria Parasite Detection in Thin Blood Smear Images. PeerJ, 6, e4568. https://doi.org/10.7717/peerj.4568 
[81] Nielsen, A., Hansen, M.B., Tietze, A. and Mouridsen, K. (2018) Prediction of Tissue Outcome and Assessment of Treatment Effect in Acute Ischemic Stroke Using Deep Learning. Stroke, 49, 1394-1401. https://doi.org/10.1161/STROKEAHA.117.019740

[82] Lee, H.C., Ryu, H.G., Chung, E.J. and Jung, C.W. (2018) Prediction of Bispectral Index during Target-Controlled Infusion of Propofol and Remifentanil: A Deep Learning Approach. Anesthesiology, 128, 492-501. https://doi.org/10.1097/ALN.0000000000001892

[83] Zeng, L.L., Wang, H., Hu, P., Yang, B., Pu, W., Shen, H., Chen, X., Liu, Z., Yin, H., Tan, Q., Wang, K. and Hu, D. (2018) Multi-Site Diagnostic Classification of Schizophrenia Using Discriminant Deep Learning with Functional Connectivity MRI. EBioMedicine, 30, 74-85. https://doi.org/10.1016/j.ebiom.2018.03.017

[84] Ghesu, F.C., Georgescu, B., Zheng, Y., Hornegger, J. and Comaniciu, D. (2015) Marginal Space Deep Learning: Efficient Architecture for Detection in Volumetric Image Data. In: Navab, N., Hornegger, J., Wells, W. and Frangi, A., Eds., Medical Image Computing and Computer-Assisted Intervention-MICCAI 2015. MICCAI 2015. Lecture Notes in Computer Science, Vol. 9349, Springer, Cham, 710-718. https://doi.org/10.1007/978-3-319-24553-9 87

[85] Anthimopoulos, M., Christodoulidis, S., Ebner, L., Christe, A. and Mougiakakou, S. (2016) Lung Pattern Classification for Interstitial Lung Diseases Using a Deep Convolutional Neural Network. IEEE Transactions on Medical Imaging, 35, 1207 1216. https://doi.org/10.1109/TMI.2016.2535865

[86] Yasaka, K., Akai, H., Kunimatsu, A., Abe, O. and Kiryu, S. (2018) Liver Fibrosis: Deep Convolutional Neural Network for Staging by Using Gadoxetic Acid-Enhanced Hepatobiliary Phase MR Images. Radiology, 287, 146-155. https://doi.org/10.1148/radiol.2017171928

[87] Cho, K., Van Merriënboer, B., Gulcehre, C., Bahdanau, D., Bougares, F., Schwenk, H. and Bengio, Y. (2014) Learning Phrase Representations Using RNN Encoder-Decoder for Statistical Machine Translation. Proceedings of the 2014 Conference on Empirical Methods in Natural Language Processing (EMNLP), Doha, October 2014, 1724-1734. https://doi.org/10.3115/v1/D14-1179

[88] Leibig, C., Allken, V., Ayhan, M.S., Berens, P. and Wahl, S. (2017) Leveraging Uncertainty Information from Deep Neural Networks for Disease Detection. Scientific Reports, 7, Article No. 17816. https://doi.org/10.1038/s41598-017-17876-Z

[89] Kooi, T., Litjens, G., van Ginneken, B., Gubern-Mérida, A., Sánchez, C.I., Mann, R., den Heeten, A. and Karssemeijer, N. (2017) Large Scale Deep Learning for Computer Aided Detection of Mammographic Lesions. Medical Image Analysis, 35, 303-312. https://doi.org/10.1016/j.media.2016.07.007

[90] Schuster, M. and Paliwal, K.K. (1997) Bidirectional Recurrent Neural Networks. IEEE Transactions on Signal Processing, 45, 2673-2681. https://doi.org/10.1109/78.650093

[91] Kim, E.K., Kim, H.E., Han, K., Kang, B.J., Sohn, Y.M., Woo, O.H. and Lee, C.W. (2018) Applying Data-Driven Imaging Biomarker in Mammography for Breast Cancer Screening: Preliminary Study. Scientific Reports, 8, Article No. 2762. https://doi.org/10.1038/s41598-018-21215-1

[92] Heinsfeld, A.S., Franco, A.R., Craddock, R.C., Buchweitz, A. and Meneguzzi, F. (2018) Identification of Autism Spectrum Disorder Using Deep Learning and the ABIDE Dataset. NeuroImage: Clinical, 17, 16-23. https://doi.org/10.1016/j.nicl.2017.08.017

[93] Suk, H.I., Lee, S.W. and Shen, D. (2014) Hierarchical Feature Representation and 
Multimodal Fusion with Deep Learning for AD/MCI Diagnosis. NeuroImage, 101, 569-582. https://doi.org/10.1016/j.neuroimage.2014.06.077

[94] Hinton, G., Vinyals, O. and Dean, J. (2015) Distilling the Knowledge in a Neural Network. arXiv: 1503.02531

[95] van Grinsven, M.J., van Ginneken, B., Hoyng, C.B., Theelen, T. and Sanchez, C.I. (2016) Fast Convolutional Neural Network Training Using Selective Data Sampling: Application to Hemorrhage Detection in Color Fundus Images. IEEE Transactions on Medical Imaging, 35, 1273-1284. https://doi.org/10.1109/TMI.2016.2526689

[96] Wang, J., Yang, X., Cai, H., et al. (2016) Discrimination of Breast Cancer with Microcalcifications on Mammography by Deep Learning. Scientific Reports, 6, Article No. 27327. https://doi.org/10.1038/srep27327

[97] Kooi, T., van Ginneken, B., Karssemeijer, N. and den Heeten, A. (2017) Discriminating Solitary Cysts from Soft Tissue Lesions in Mammography Using a Pretrained Deep Convolutional Neural Network. Medical Physics, 44, 1017-1027. https://doi.org/10.1002/mp.12110

[98] Fu, H., Cheng, J., Xu, Y., Zhang, C., Wong, D.W.K., Liu, J. and Cao, X. (2018) DiscAware Ensemble Network for Glaucoma Screening from Fundus Image. IEEE Transactions on Medical Imaging, 37, 2493-2501. https://doi.org/10.1109/TMI.2018.2837012

[99] Yu, C., Yang, S., Kim, W., Jung, J., Chung, K.Y., Lee, S.W. and Oh, B. (2018) Acral Melanoma Detection Using a Convolutional Neural Network for Dermoscopy Images. PLoS ONE, 13, e0193321. https://doi.org/10.1371/journal.pone.0193321

[100] Topol, E. (2019) Deep Medicine: How Artificial Intelligence Can Make Healthcare Human Again. Basic Books, Hachette, UK.

[101] Wang, J., Ding, H., Bidgoli, F.A., Zhou, B., Iribarren, C., Molloi, S. and Baldi, P. (2017) Detecting Cardiovascular Disease from Mammograms with Deep Learning. IEEE Transactions on Medical Imaging, 36, 1172-1181. https://doi.org/10.1109/TMI.2017.2655486

[102] Rumelhart, D.E., Hinton, G.E. and Williams, R.J. (1985) Learning Internal Representations by Error Propagation. California Univ San Diego La Jolla Inst for Cognitive Science.

[103] Iakovidis, D.K., Georgakopoulos, S.V., Vasilakakis, M., Koulaouzidis, A. and Plagianakos, V.P. (2018) Detecting and Locating Gastrointestinal Anomalies Using Deep Learning and Iterative Cluster Unification. IEEE Transactions on Medical Imaging, 37, 2196-2210. https://doi.org/10.1109/TMI.2018.2837002

[104] van der Burgh, H.K., Schmidt, R., Westeneng, H.J., de Reus, M.A., van den Berg, L.H. and van den Heuvel, M.P. (2016) Deep Learning Predictions of Survival Based on MRI in Amyotrophic Lateral Sclerosis. NeuroImage: Clinical, 13, 361-369. https://doi.org/10.1016/j.nicl.2016.10.008

[105] Lee, C.S., Baughman, D.M. and Lee, A.Y. (2017) Deep Learning Is Effective for the Classification of OCT Images of Normal Versus Age-Related Macular Degeneration. Ophthalmology Retina, 1, 322-327. https://doi.org/10.1016/j.oret.2016.12.009

[106] Hsieh, Y.J., Tseng, H.C., Chin, C.L., Shao, Y.H. and Tsai, T.Y. (2020) Based on DICOM RT Structure and Multiple Loss Function Deep Learning Algorithm in Organ Segmentation of Head and Neck Image. In: Lin, K.P., Magjarevic, R. and de Carvalho, P., Eds., Future Trends in Biomedical and Health Informatics and $C y$ bersecurity in Medical Devices. ICBHI 2019. IFMBE Proceedings, Vol. 74, Springer, Cham, 428-435. https://doi.org/10.1007/978-3-030-30636-6 58

[107] Ngo, T.A., Lu, Z. and Carneiro, G. (2017) Combining Deep Learning and Level Set 
for the Automated Segmentation of the Left Ventricle of the Heart from Cardiac Cine Magnetic Resonance. Medical Image Analysis, 35, 159-171. https://doi.org/10.1016/j.media.2016.05.009

[108] Zhang, J., Xia, Y., Wu, Q. and Xie, Y.T. (2017) Classification of Medical Images and Illustrations in the Biomedical Literature Using Synergic Deep Learning. arXiv: 1706.09092

[109] Araújo, T., Aresta, G., Castro, E., Rouco, J., Aguiar, P., Eloy, C., Polónia, A. and Campilho, A. (2017) Classification of Breast Cancer Histology Images Using Convolutional Neural Networks. PLoS ONE, 12, e0177544.

https://doi.org/10.1371/journal.pone.0177544

[110] Han, Z., Wei, B., Zheng, Y., Yin, Y., Li, K. and Li, S. (2017) Breast Cancer Multi-Classification from Histopathological Images with Structured Deep Learning Model. Scientific Reports, 7, Article No. 4172. https://doi.org/10.1038/s41598-017-04075-Z

[111] Zhang, X., Yu, F.X., Chang, S.-F. and Wang, S.J. (2015) Deep Transfer Network: Unsupervised Domain Adaptation. arXiv: 1503.00591

[112] Hutchinson, B., Deng, L. and Yu, D. (2013) Tensor Deep Stacking Networks. IEEE Transactions on Pattern Analysis and Machine Intelligence, 35, 1944-1957. https://doi.org/10.1109/TPAMI.2012.268

[113] Hjelm, R.D., Fedorov, A., Lavoie-Marchildon, S., Grewal, K., Bachman, P., Trischler, A. and Bengio, Y. (2018) Learning Deep Representations by Mutual Information Estimation and Maximization. arXiv: 1808.06670

[114] Alom, M.Z., Yakopcic, C., Nasrin, M.S., Taha, T.M. and Asari, V.K. (2019) Breast Cancer Classification from Histopathological Images with Inception Recurrent Residual Convolutional Neural Network. Journal of Digital Imaging, 32, 605-617. https://doi.org/10.1007/s10278-019-00182-7

[115] Tiulpin, A., Thevenot, J., Rahtu, E., Lehenkari, P. and Saarakkala, S. (2018) Automatic Knee Osteoarthritis Diagnosis from Plain Radiographs: A Deep Learning-Based Approach. Scientific Reports, 8, Article No. 1727. https://doi.org/10.1038/s41598-018-20132-7

[116] Lee, J. and Nishikawa, R.M. (2018) Automated Mammographic Breast Density Estimation Using a Fully Convolutional Network. Medical Physics, 45, 1178-1190. https://doi.org/10.1002/mp.12763

[117] Esses, S.J., Lu, X., Zhao, T., Shanbhogue, K., Dane, B., Bruno, M. and Chandarana, H. (2018) Automated Image Quality Evaluation of T2-Weighted Liver MRI Utilizing Deep Learning Architecture. Journal of Magnetic Resonance Imaging, 47, 723 728. https://doi.org/10.1002/jmri.25779

[118] Serj, M.F., Lavi, B., Hoff, G. and Valls, D.P. (2018) A Deep Convolutional Neural Network for Lung Cancer Diagnostic. arXiv: 1804.08170

[119] Du, X., et al. (2018) Articulated Multi-Instrument 2-D Pose Estimation Using Fully Convolutional Networks. IEEE Transactions on Medical Imaging, 37, 1276-1287. https://doi.org/10.1109/TMI.2017.2787672

[120] Han, S.S., Park, G.H., Lim, W., Kim, M.S., Na, J.I., Park, I. and Chang, S.E. (2018) Deep Neural Networks Show an Equivalent and Often Superior Performance to Dermatologists in Onychomycosis Diagnosis: Automatic Construction of Onychomycosis Datasets by Region-Based Convolutional Deep Neural Network. PLoS ONE, 13, e0191493. https://doi.org/10.1371/journal.pone.0191493

[121] Kim, K.H., Choi, S.H. and Park, S.H. (2018) Improving Arterial Spin Labeling by Using Deep Learning. Radiology, 287, 658-666. 
https://doi.org/10.1148/radiol.2017171154

[122] Song, Y., Zhang, L., Chen, S., Ni, D., Lei, B. and Wang, T. (2015) Accurate Segmentation of Cervical Cytoplasm and Nuclei Based on Multiscale Convolutional Network and Graph Partitioning. IEEE Transactions on Biomedical Engineering, 62, 2421-2433. https://doi.org/10.1109/TBME.2015.2430895

[123] Haryanto, T., Wasito, I. and Suhartanto, H. (2017) Convolutional Neural Network (CNN) for Gland Images Classification. 2017 11th International Conference on Information \& Communication Technology and System (ICTS), Surabaya, 31-31 October 2017, 55-60. https://doi.org/10.1109/ICTS.2017.8265646

[124] Cao, H., Bernard, S., Heutte, L. and Sabourin, R. (2018) Improve the Performance of Transfer Learning without Fine-Tuning Using Dissimilarity-Based Multi-View Learning for Breast Cancer Histology Images. In: Campilho, A., Karray, F. and ter Haar Romeny, B., Eds., Image Analysis and Recognition. ICIAR 2018. Lecture Notes in Computer Science, Vol. 10882, Springer, Cham, 779-787.

https://doi.org/10.1007/978-3-319-93000-8 88

[125] Luo, X., Mori, K. and Peters, T.M. (2018) Advanced Endoscopic Navigation: Surgical Big Data, Methodology, and Applications. Annual Review of Biomedical Engineering, 20, 221-251. https://doi.org/10.1146/annurev-bioeng-062117-120917

[126] Xiao, C., Choi, E. and Sun, J. (2018) Opportunities and Challenges in Developing Deep Learning Models Using Electronic Health Records Data: A Systematic Review. Journal of the American Medical Informatics Association, 25, 1419-1428. https://doi.org/10.1093/jamia/ocy068

[127] Shickel, B., Tighe, P.J., Bihorac, A. and Rashidi, P. (2018) Deep EHR: A Survey of Recent Advances in Deep Learning Techniques for Electronic Health Record (EHR) Analysis. IEEE Journal of Biomedical and Health Informatics, 22, 1589-1604. https://doi.org/10.1109/JBHI.2017.2767063

[128] Karkra, S., Singh, P. and Kaur, K. (2019) Convolution Neural Network: A Shallow Dive into Deep Neural Net Technology. International Journal of Recent Technology and Engineering (IJRTE), 8, 487-495.

[129] Ranschaert, E.R., Morozov, S. and Algra, P.R. (2019) Artificial Intelligence in Medical Imaging: Opportunities, Applications and Risks. Springer, Berlin. https://doi.org/10.1007/978-3-319-94878-2

[130] Tsang, G., Xie, X. and Zhou, S.M. (2020) Harnessing the Power of Machine Learning in Dementia Informatics Research: Issues, Opportunities, and Challenges. IEEE Reviews in Biomedical Engineering, 13, 113-129. https://doi.org/10.1109/RBME.2019.2904488

[131] Haryanto, T., Suhartanto, H., Murni, A. and Kusmardi, K. (2019) Strategies to Improve Performance of Convolutional Neural Network on Histopathological Images Classification. 2019 International Conference on Advanced Computer Science and information Systems (ICACSIS), Bali, Indonesia, 12-13 October 2019, 125-132. https://doi.org/10.1109/ICACSIS47736.2019.8979740

[132] Das, A., Nair, M.S. and Peter, S.D. (2020) Computer-Aided Histopathological Image Analysis Techniques for Automated Nuclear Atypia Scoring of Breast Cancer: A Review. Journal of Digital Imaging, 33, 1091-1121. https://doi.org/10.1007/s10278-019-00295-Z

[133] Tang, Y., Wang, X., Harrison, A.P., Lu, L., Xiao, J. and Summers, R.M. (2018) Attention-Guided Curriculum Learning for Weakly Supervised Classification and Localization of Thoracic Diseases on Chest Radiographs. In: Shi, Y., Suk, H.I. and Liu, M., Eds., Machine Learning in Medical Imaging. MLMI2018. Lecture Notes in 
Computer Science, Vol. 11046, Springer, Cham, 249-258. https://doi.org/10.1007/978-3-030-00919-9 29

[134] Goodfellow, I.J., Pouget-Abadie, J., Mirza, M., Xu, B., Warde-Farley, D., Ozair, S., Courville, A. and Bengio, Y. (2014) Generative Adversarial Nets. Proceedings of the 27 th International Conference on Neural Information Processing Systems, 2, 2672-2680.

[135] Hoffman, J., Tzeng, E., Park, T., Zhu, J.Y., Isola, P., Saenko, K., et al. (2018) Cycada: Cycle-Consistent Adversarial Domain Adaptation. Proceedings of the 35 th International Conference on Machine Learning, PMLR, 80, 1989-1998.

[136] Long, M., Zhu, H., Wang, J. and Jordan, M.I. (2016) Unsupervised Domain Adaptation with Residual Transfer Networks. arXiv: 1602.04433

[137] Tzeng, E., Hoffman, J., Saenko, K. and Darrell, T. (2017) Adversarial Discriminative Domain Adaptation. Proceedings of the IEEE Conference on Computer Vision and Pattern Recognition, Honolulu, HI, 21-26 July 2017, 2962-2971. https://doi.org/10.1109/CVPR.2017.316

[138] Luo, Y., Zheng, L., Guan, T., Yu, J. and Yang, Y. (2019) Taking a Closer Look at Domain Shift: Category-Level Adversaries for Semantics Consistent Domain Adaptation. Proceedings of the IEEE/CVF Conference on Computer Vision and Pattern Recognition, Long Beach, CA, 15-20 June 2019, 2502-2511. https://doi.org/10.1109/CVPR.2019.00261

[139] Tsai, Y.H., Hung, W.C., Schulter, S., Sohn, K., Yang, M.H. and Chandraker, M. (2018) Learning to Adapt Structured Output Space for Semantic Segmentation. Proceedings of the IEEE Conference on Computer Vision and Pattern Recognition, 7472-7481. https://doi.org/10.1109/CVPR.2018.00780

[140] Liu, D., Zhang, D., Song, Y., Zhang, F., O’Donnell, L., Huang, H., Chen, M. and Cai, W. (2021) PDAM: A Panoptic-Level Feature Alignment Framework for Unsupervised Domain Adaptive Instance Segmentation in Microscopy Images. IEEE Transactions on Medical Imaging, 40, 154-165. https://doi.org/10.1109/TMI.2020.3023466

[141] Ghafoorian, M., et al. (2017) Transfer Learning for Domain Adaptation in MRI: Application in Brain Lesion Segmentation. In: Descoteaux, M., Maier-Hein, L., Franz, A., Jannin, P., Collins, D. and Duchesne, S., Eds., Medical Image Computing and Computer Assisted Intervention-MICCAI 2017. MICCAI 2017. Lecture Notes in Computer Science, Vol. 10435, Springer, Cham, 516-524.

[142] Jiang, J., Hu, Y.C., Tyagi, N., Zhang, P., Rimner, A., Mageras, G.S., Deasy, J.O. and Veeraraghavan, H. (2018) Tumor-Aware, Adversarial Domain Adaptation from CT to MRI for Lung Cancer Segmentation. In: Frangi, A., Schnabel, J., Davatzikos, C., Alberola-López, C. and Fichtinger, G., Eds., Medical Image Computing and Computer Assisted Intervention-MICCAI 2018. MICCAI 2018. Lecture Notes in Computer Science, Vol. 11071, Springer, Cham, 777-785.

https://doi.org/10.1007/978-3-030-00934-2 86

[143] Chen, C., Dou, Q., Chen, H. and Heng, P.A. (2018) Semantic-Aware Generative Adversarial Nets for Unsupervised Domain Adaptation in Chest X-Ray Segmentation. In: Shi, Y., Suk, H.I. and Liu, M., Eds., Machine Learning in Medical Imaging. MLMI 2018. Lecture Notes in Computer Science, Vol. 11046, Springer, Cham, 143-151. https://doi.org/10.1007/978-3-030-00919-9 17

[144] Yang, J., Dvornek, N.C., Zhang, F., Chapiro, J., Lin, M. and Duncan, J.S. (2019) Unsupervised Domain Adaptation via Disentangled Representations: Application to Cross-Modality Liver Segmentation. In: Shen, D., et al., Eds., Medical Image Computing and Computer Assisted Intervention-MICCAI 2019. MICCAI 2019. 
Lecture Notes in Computer Science, Vol. 11765, Springer, Cham, 255-263. https://doi.org/10.1007/978-3-030-32245-8 29

[145] Zhang, C., Wu, S., Lu, Z., Shen, Y., Wang, J., Huang, P., Lou, J., Liu, C., Xing, L., Zhang, J., Xue, J. and Li, D. (2020) Hybrid Adversarial-Discriminative Network for Leukocyte Classification in Leukemia. Medical Physics, 47, 3732-3744. https://doi.org/10.1002/mp.14144

[146] Li, C.Y., Liang, X., Hu, Z. and Xing, E.P. (2019) Knowledge-Driven Encode, Retrieve, Paraphrase for Medical Image Report Generation. Proceedings of the AAAI Conference on Artificial Intelligence, 33, 6666-6673. https://doi.org/10.1609/aaai.v33i01.33016666

[147] Wang, Z., Zhang, J., Feng, J. and Chen, Z. (2014) Knowledge Graph and Text Jointly Embedding. Proceedings of the 2014 Conference on Empirical Methods in Natural Language Processing (EMNLP), Doha, October 2014, 1591-1601. https://doi.org/10.3115/v1/D14-1167

[148] Luo, B.N., Shen, J., Cheng, S.Y., Wang, Y.J. and Pantic, M. (2020) Shape Constrained Network for Eye Segmentation in the Wild. Proceedings of the IEEE/CVF Winter Conference on Applications of Computer Vision ( WACV), Snowmass, CO, 1-5 March 2020, 1952-1960. https://doi.org/10.1109/WACV45572.2020.9093483

[149] Wistuba, M., Rawat, A. and Pedapati, T. (2019) A Survey on Neural Architecture Search. arXiv: 1905. 01392.

[150] Guo, D., Jin, D., Zhu, Z., Ho, T.Y., Harrison, A.P., Chao, C.H., et al. (2020) Organ at Risk Segmentation for Head and Neck Cancer Using Stratified Learning and Neural Architecture Search. Proceedings of the IEEE/CVF Conference on Computer Vision and Pattern Recognition, Seattle, WA, 13-19 June 2020, 4222-4231. https://doi.org/10.1109/CVPR42600.2020.00428

[151] Li, S., Wei, J., Chan, H.P., Helvie, M.A., Roubidoux, M.A., Lu, Y., Zhou, C., Hadjiiski, L.M. and Samala, R.K. (2018) Computer-Aided Assessment of Breast Density: Comparison of Supervised Deep Learning and Feature-Based Statistical Learning. Physics in Medicine \& Biology, 63, Article ID: 025005. https://doi.org/10.1088/1361-6560/aa9f87

[152] Carneiro, G., Nascimento, J.C. and Freitas, A. (2012) The Segmentation of the Left Ventricle of the Heart From Ultrasound Data Using Deep Learning Architectures and Derivative-Based Search Methods. IEEE Transactions on Image Processing, 21, 968-982. https://doi.org/10.1109/TIP.2011.2169273

[153] Xue, Y., Zhang, R., Deng, Y., Chen, K. and Jiang, T. (2017) A Preliminary Examination of the Diagnostic Value of Deep Learning in Hip Osteoarthritis. PLoS ONE, 12, e0178992. https://doi.org/10.1371/journal.pone.0178992

[154] Chen, C.-M., Huang, Y.-S., Fang, P.-W., Liang, C.-W. and Chang, R.-F. (2020) A Computer-Aided Diagnosis System for Differentiation and Delineation of Malignant Regions on Whole-Slide Prostate Histopathology Image Using Spatial Statistics and Multidimensional DenseNet. Medical Physics, 47, 1021-1033. https://doi.org/10.1002/mp.13964

[155] Quellec, G., Charrière, K., Boudi, Y., Cochener, B. and Lamard, M. (2017) Deep Image Mining for Diabetic Retinopathy Screening. Medical Image Analysis, 39, 178-193. https://doi.org/10.1016/j.media.2017.04.012

[156] Saha, S.K., Fernando, B., Cuadros, J., Xiao, D. and Kanagasingam, Y. (2018) Automated Quality Assessment of Colour Fundus Images for Diabetic Retinopathy Screening in Telemedicine. Journal of Digital Imaging, 31, 869-878. https://doi.org/10.1007/s10278-018-0084-9 
[157] Das, A., Rad, P., Choo, K.R., Nouhi, B., Lish, J. and Martel, J. (2019) Distributed Machine Learning Cloud Teleophthalmology IoT for Predicting AMD Disease Progression. Future Generation Computer Systems, 93, 486-498. https://doi.org/10.1016/j.future.2018.10.050

[158] Kim, Y D., Noh, K.J., Byun, S.J., et al. (2020) Effects of Hypertension, Diabetes, and Smoking on Age and Sex Prediction from Retinal Fundus Images. Scientific Reports, 10, Article No. 4623. https://doi.org/10.1038/s41598-020-61519-9

[159] Betancur, J., Commandeur, F., Motlagh, M., Sharir, T., Einstein, A.J., et al. (2018) Deep Learning for Prediction of Obstructive Disease from Fast Myocardial Perfusion SPECT: A Multicenter Study. JACC: Cardiovascular Imaging, 11, 1654-1663. https://doi.org/10.1016/j.jcmg.2018.01.020

[160] Chaudhari, A.S., Fang, Z., Kogan, F., Wood, J., Stevens, K.J., Gibbons, E.K., Lee, J.H., Gold, G.E. and Hargreaves, B.A. (2018) Super-Resolution Musculoskeletal MRI Using Deep Learning. Magnetic Resonance in Medicine, 80, 2139-2154. https://doi.org/10.1002/mrm.27178

[161] Ning, Z., Luo, J., Li, Y., Han, S., Feng, Q., Xu, Y., Chen, W., Chen, T. and Zhang, Y. (2019) Pattern Classification for Gastrointestinal Stromal Tumors by Integration of Radiomics and Deep Convolutional Features. IEEE Journal of Biomedical and Health Informatics, 23, 1181-1191. https://doi.org/10.1109/JBHI.2018.2841992

[162] Khosravan, N., Celik, H., Turkbey, B., Jones, E.C., Wood, B. and Bagci, U. (2019) A Collaborative Computer Aided Diagnosis (C-CAD) System with Eye-Tracking, Sparse Attentional Model, and Deep Learning. Medical Image Analysis, 51, 101-115. https://doi.org/10.1016/j.media.2018.10.010

[163] Jang, R., Kim, N., Jang, M., Lee, K.H., Lee, S.M., Lee, K.H., Noh, H.N. and Seo, J.B. (2020) Assessment of the Robustness of Convolutional Neural Networks in Labeling Noise by Using Chest X-Ray Images from Multiple Centers. JMIR Medical Informatics, 8, e18089. https://doi.org/10.2196/18089

[164] Cheng, J.Z., Ni, D., Chou, Y.H., Qin, J., Tiu, C.M., Chang, Y.C., Huang, C.S., Shen, D. and Chen, C.M. (2016) Computer-Aided Diagnosis with Deep Learning Architecture: Applications to Breast Lesions in US Images and Pulmonary Nodules in CT Scans. Scientific Reports, 6, Article No. 24454.

https://doi.org/10.1038/srep24454

[165] Song, Y., Zhang, Y.D., Yan, X., Liu, H., Zhou, M., Hu, B. and Yang, G. (2018) Computer-Aided Diagnosis of Prostate Cancer Using a Deep Convolutional Neural Network from Multiparametric MRI. Journal of Magnetic Resonance Imaging, 48, 1570-1577. https://doi.org/10.1002/jmri.26047

[166] Sujit, S.J., Coronado, I., Kamali, A., Narayana, P.A. and Gabr, R.E. (2019) Automated Image Quality Evaluation of Structural Brain MRI Using an Ensemble of Deep Learning Networks. Journal of Magnetic Resonance Imaging, 50, 1260-1267. https://doi.org/10.1002/jmri.26693

[167] Dar, S.U.H., Yurt, M., Shahdloo, M., Ildız, M.E. and Çukur, T. (2018) Synergistic Reconstruction and Synthesis via Generative Adversarial Networks for Accelerated Multi-Contrast MRI. Computer Vision and Pattern Recognition. arXiv: 1805.10704. 